Prepared for the Washington State Department of Ecology

\title{
Sediment Toxicity Test Results for the Urban Waters Study 2010, Bellingham Bay, Washington
}

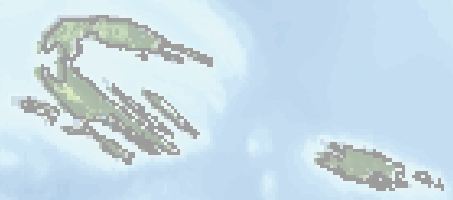

Data Series 591
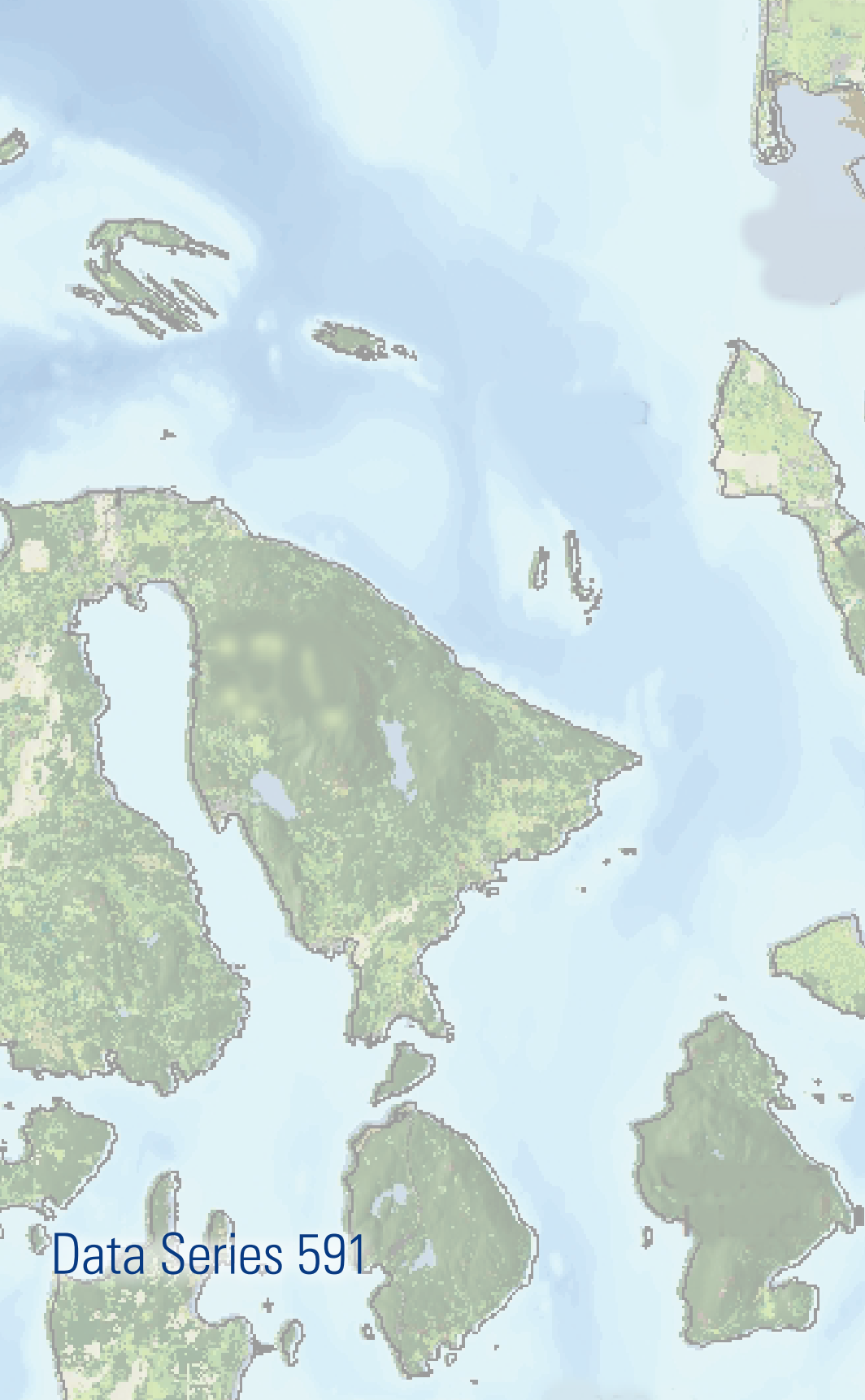

Interior
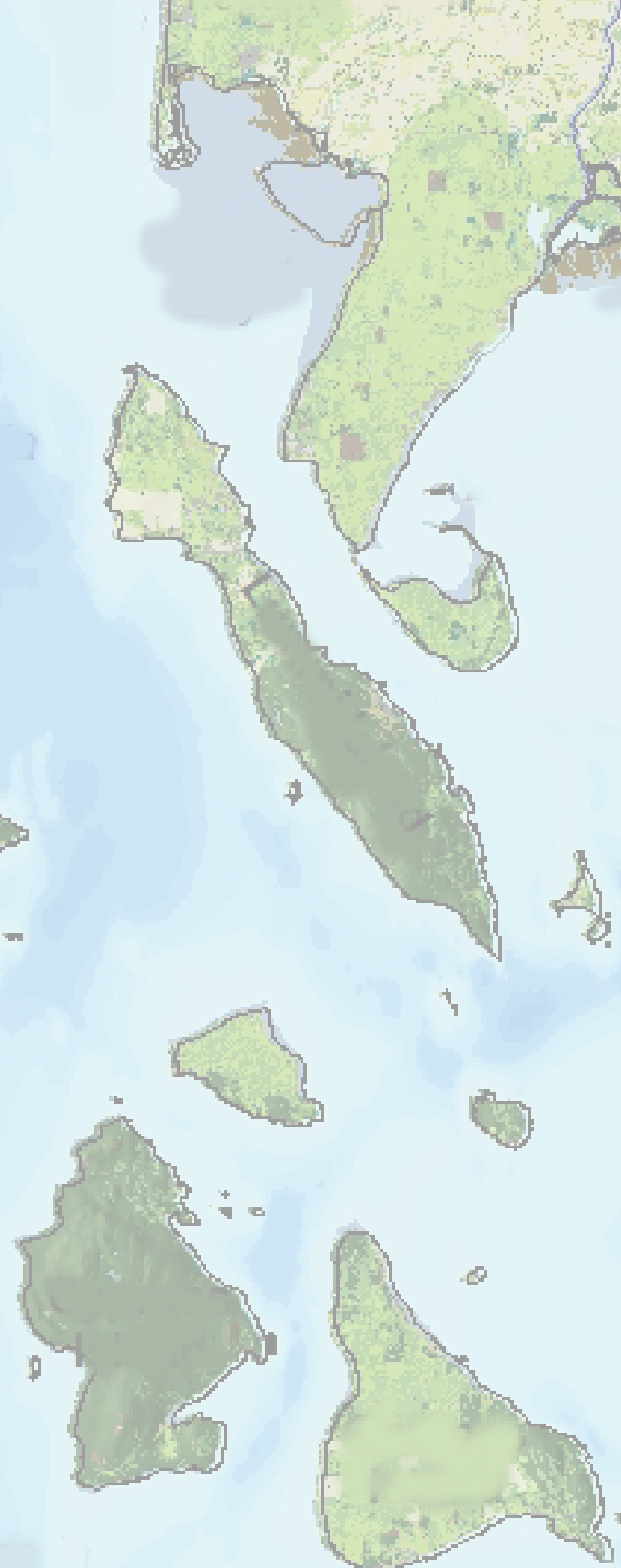
Cover: Map of Bellingham Bay and nearby Samish Bay, Padilla Bay and the Rosario Strait, Washington. Map by S. Weakland, Washington State Department of Ecology, 2010. 


\title{
Sediment Toxicity Test Results for the Urban Waters Study 2010, Bellingham Bay, Washington
}

\author{
By James M. Biedenbach
}

Prepared for the Washington State Department of Ecology

Data Series 591 


\section{U.S. Department of the Interior \\ KEN SALAZAR, Secretary \\ U.S. Geological Survey \\ Marcia K. McNutt, Director}

\section{U.S. Geological Survey, Reston, Virginia: 2011}

For more information on the USGS - the Federal source for science about the Earth, its natural and living resources, natural hazards, and the environment, visit http://www.usgs.gov or call 1-888-ASK-USGS.

For an overview of USGS information products, including maps, imagery, and publications, visit http://www.usgs.gov/pubprod

To order this and other USGS information products, visit http://store.usgs.gov

Any use of trade, product, or firm names is for descriptive purposes only and does not imply endorsement by the U.S. Government.

Although this report is in the public domain, permission must be secured from the individual copyright owners to reproduce any copyrighted materials contained within this report.

Suggested citation:

Biedenbach, J.M., 2011, Sediment toxicity test results for the Urban Waters Study 2010, Bellingham Bay, Washington: U.S. Geological Survey Data Series 591, 65 p. plus 9 appendixes. 


\section{Acknowledgments}

The author would like to thank Dr. Kim Withers, Kim McGlaun and Alex Rafalski of the Texas

A\&M University-Corpus Christi's Center for Coastal Studies for their assistance in completing the toxicity testing and water-quality analysis. 


\section{Contents}

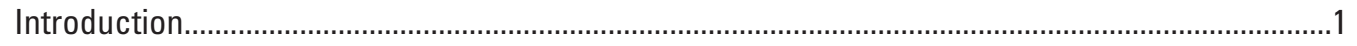

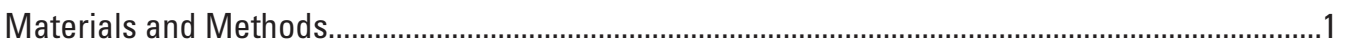

Sediment Sample Receipt and Tracking ….......................................................................

Toxicity Testing .....................................................................................................................

Sediment Pore-Water Extraction Procedure ..................................................................

Toxicity Testing with Sea Urchins .....................................................................................

Sea Urchin Data Analysis.............................................................................................

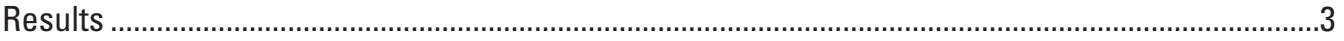

Pore-Water Quality Measurements.....................................................................................

Sea Urchin Toxicity Testing ......................................................................................................

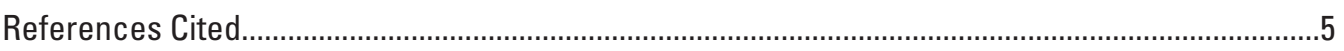

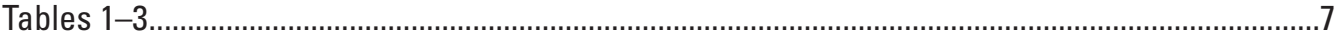

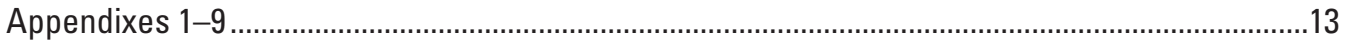

\section{Figures}

1. Map showing station locations of sediments and toxicity testing results for the 2010 Urban Waters study in Bellingham Bay, Washington

\section{Tables}

1. Water-quality measurements after salinity adjustment and original salinity of sediment pore-water samples collected for the 2010 Urban Waters study in Bellingham Bay, Washington..

2. Sea urchin fertilization test raw data and means for sediment pore-water samples in test one of the Urban Waters 2010 study in Bellingham Bay, Washington.....

3. Sea urchin fertilization test raw data and means for sediment pore-water samples in test two of the Urban Waters 2010 study in Bellingham Bay, Washington 


\section{Conversion Factors}

SI to Inch/Pound

\begin{tabular}{lcl}
\hline \multicolumn{1}{c}{ Multiply } & \multicolumn{1}{c}{ By } & \multicolumn{1}{c}{ To obtain } \\
\hline liter $(\mathrm{L})$ & Volume & \\
liter $(\mathrm{L})$ & 33.82 & ounce, fluid (fl. oz) \\
liter $(\mathrm{L})$ & 2.113 & pint (pt) \\
liter $(\mathrm{L})$ & 1.057 & quart (qt) \\
liter $(\mathrm{L})$ & .2642 & gallon (gal) \\
milliliters $(\mathrm{mL})$ & 61.02 & cubic inch (in3) \\
microliters $(\mu \mathrm{L})$ & .3382 & ounce, fluid (fl. oz) \\
& .00003382 & ounce, fluid (fl. oz) \\
\hline gram $(\mathrm{g})$ & Mass & \\
milligrams $(\mathrm{mg})$ & 0.03527 & ounce, avoirdupois $(\mathrm{oz})$ \\
micrograms $(\mu \mathrm{g})$ & .00003527 & ounce, avoirdupois $(\mathrm{oz})$ \\
& .00000003527 & ounce, avoirdupois $(\mathrm{oz})$ \\
\hline micrometer $(\mu \mathrm{m})$ & Length & \\
\hline
\end{tabular}

Temperature in degrees Celsius $\left({ }^{\circ} \mathrm{C}\right)$ may be converted to degrees Fahrenheit $\left({ }^{\circ} \mathrm{F}\right)$ as follows:

$$
{ }^{\circ} \mathrm{F}=\left(1.8 \times{ }^{\circ} \mathrm{C}\right)+32
$$

Concentrations of chemical constituents in water are given either in milligrams per liter (mg/L) or micrograms per liter $(\mu \mathrm{g} / \mathrm{L})$.

Salinities are given in parts per thousand $(\%)$ equivalent to grams per liter $(\mathrm{g} / \mathrm{L})$. 



\title{
Sediment Toxicity Test Results for the Urban Waters Study 2010, Bellingham Bay, Washington
}

\author{
By James M. Biedenbach
}

\section{Introduction}

The Washington Department of Ecology annually determines the quality of recently deposited sediments in Puget Sound as a part of Ecology's Urban Waters Initiative. The annual sediment quality studies use the Sediment Quality Triad (SQT) approach, thus relying on measures of chemical contamination, toxicity, and benthic in-faunal effects (Chapman, 1990). Since 2002, the studies followed a rotating sampling scheme, each year sampling a different region of the greater Puget Sound Basin. During the annual studies, samples are collected in locations selected with a stratified-random design, patterned after the designs previously used in baseline surveys completed during 1997-1999 (Long and others, 2003; Wilson and Partridge, 2007).

Sediment samples were collected by personnel from the Washington Department of Ecology, in June of 2010 and shipped to the U. S. Geological Survey (USGS) laboratory in Corpus Christi, Texas (not shown), where the tests were performed. Sediment pore water was extracted with a pneumatic apparatus and was stored frozen. Just before testing, water-quality measurements were made and salinity adjusted, if necessary. Tests were performed on a dilution series of each sample consisting of 100-, 50-, and 25-percent pore-water concentrations.

The specific objectives of this study were to:

- Extract sediment pore water from a total of 30 sediment samples from the Bellingham Bay, Washington area within a day of receipt of the samples.

- Measure water-quality parameters (salinity, dissolved oxygen, $\mathrm{pH}$, sulfide, and ammonia) of thawed porewater samples before testing and adjust salinity, temperature and dissolved oxygen, if necessary, to obtain optimal ranges for the test species.

- Conduct the fertilization toxicity test with pore water using sea urchin (Stronylocentrotus purpuratus) (S. purpuratus) gametes.

- Perform quality control assays with reference pore water, dilution blanks and a positive control dilution series with sodium dodecyl sulfate (SDS) in conjunction with each test.
- Determine which samples caused a significant decrease in percent fertilization success relative to the negative control.

\section{Materials and Methods}

\section{Sediment Sample Receipt and Tracking}

Surficial sediment samples were collected from 30 stations in areas throughout Bellingham Bay, Washington. Samples were placed in precleaned 1-gallon high density polyethylene containers, chilled, and shipped in insulated coolers with blue ice. Samples were received by the USGS in Corpus Christi, Texas, the day following shipment. Shipments were accompanied by sample tracking sheets, and samples were logged into laboratory sample tracking systems (appendix 1). All pore-water samples were extracted within 6 days from the time of field collection of sediment, and within 30 hours of arrival at the Corpus Christi laboratory (appendix 2).

\section{Toxicity Testing}

\section{Sediment Pore-Water Extraction Procedure}

Approximately 500 milliliters $(\mathrm{mL})$ of pore water was extracted from each sediment sample using a pneumatic extraction device. The extractor is made of polyvinyl chloride (PVC) and uses a 5-micrometer $(\mu \mathrm{m})$ polyester filter. It is the same device used in previous sediment quality assessment surveys (Carr and Chapman, 1992, 1995; Carr and others, 1996a, 1996b, 1997, 2001). The apparatus and extraction procedures are detailed in CERC SOP P.649 (appendix 3). After extraction, the pore-water samples were centrifuged in polycarbonate bottles at 1,200 times gravity (g) for 20 minutes to remove any suspended particulate material; the supernatant was collected, divided into three precleaned sample bottles, and frozen at negative 20 degrees Celsius $\left({ }^{\circ} \mathrm{C}\right)$.

Two days before conducting a toxicity test, one bottle from each station was moved from the freezer to a refrigerator kept at $4{ }^{\circ} \mathrm{C}$. One day before testing, samples were thawed 
and brought to room temperature in a tepid $\left(20 \pm 2{ }^{\circ} \mathrm{C}\right)$ water bath. Sample salinity was measured and adjusted to $30 \pm 1$ parts per thousand (\%o), if necessary, using purified de-ionized water or concentrated brine (appendix 4; CERC SOP P.651). Following water-quality adjustments, the samples were stored overnight at $4{ }^{\circ} \mathrm{C}$ but, were returned to $12 \pm 1{ }^{\circ} \mathrm{C}$ (incubated in an environmental chamber) immediately before the start of the toxicity tests.

On the day of the test, subsamples were collected, acclimated to room temperature and water-quality measurements (dissolved oxygen, $\mathrm{pH}$ and ammonia) were made (appendix 5; CERC SOP P.646). Additional subsamples were preserved with SAOB II reagent for sulfide measurements and refrigerated to $4{ }^{\circ} \mathrm{C}$ until the following day when they were brought to room temperature and measured (appendix 6; CERC SOP P.657). Dissolved oxygen (DO) was measured with a YSI ${ }^{\circledR}$ model 59 dissolved-oxygen meter with a YSI model 5905 BOD probe. Salinity was measured with a Reichert ${ }^{\mathbb{E}}$ temperature compensated refractometer. Sulfide $\left(\right.$ as $\left.\mathrm{S}^{-2}\right), \mathrm{pH}$ and total ammonia (expressed as nitrogen; TAN) were measured with Orion ${ }^{\circledR}$ model 290 A meters and the Thermo-Orion model 9616 silver/sulfide probe, Thermo-Orion model 9107BN low maintenance triode, and the Thermo-Orion model 5912 ammonia probe, respectively. Room and environmental chamber temperature were measured using a Fisherbrand $76 \mathrm{~mm}$ Teflon encased mercury thermometer. Un-ionized ammonia concentrations (expressed as nitrogen; UAN) were calculated for each sample using the respective salinity, temperature, $\mathrm{pH}$, and TAN values (Bowers and Bidwell, 1978). Any samples containing less than 80-percent DO saturation were aerated gently by stirring the sample on a magnetic stir plate before testing.

\section{Toxicity Testing with Sea Urchins}

S. purpuratus urchins were obtained from Marinus Scientific Inc., Los Angeles county, California (not shown). Each pore-water sample was tested in a dilution series at 100, 50 , and 25 percent of sample (after salinity adjustment) with five replicates per treatment (appendix 7; CERC SOP P.645). A pretest was conducted, which included the reference pore water, the dilution water and a limited reference toxicant dilution series, to determine the optimum sperm dilution that would produce acceptable fertilization rates in the negative control pore water, but also would produce acceptable fertilization in the dilution water and meet the USGS Corpus Christi, Texas, laboratory standard in the reference toxicant. All pretests and tests were conducted at $12{ }^{\circ} \mathrm{C}$ in a temperature controlled chamber with an exposure time of 20 minutes each for the sperm and the sperm plus eggs. Dilutions were made with $0.45-\mu \mathrm{m}$ filtered seawater collected from the ship channel in Port Aransas, Texas (not shown). A pore-water sample collected from Aransas Bay, Texas (not shown), which had been collected with a PVC corer, held refrigerated and extracted identically to the test samples, was included with each toxicity test as a negative control. This site is far removed from any known sources of contamination and has been used previously as a control site (Carr and others, 2006; Carr and Nipper, 2008; Long and others, 2008).

In addition, a dilution water blank of filtered seawater was included in each test and a brine blank (control pore water diluted to the lowest salinity measured in the test samples and subsequently increased with brine) was included in those tests that contained samples that were adjusted with brine. Finally, a dilution series test with SDS was included in each assay as a positive control to evaluate overall test sensitivity. The positive control was prepared, and diluted with the same filtered seawater used to dilute the pore waters in the dilution series described above.

\section{Sea Urchin Data Analysis}

For the fertilization test, statistical comparisons among treatments were made using analysis of varience (ANOVA) and Dunnett's one-tailed $t$-test (which controls the experimentwise error rate) on the arcsine square root transformed data with the aid of Statistical Analysis Software (SAS) (Statistical Analysis Software (SAS) Institute Incorporated, 1989). The trimmed Spearman-Karber method (Hamilton and others, 1977) with Abbott's correction (Morgan, 1992) was used to calculate 50-percent effective concentration $\left(\mathrm{EC}_{50}\right)$ values for the SDS dilution series.

Before statistical analysis, the transformed data sets were screened for equal variance using SAS/LAB ${ }^{\circledR}$ Software (Statistical Analysis Software (SAS) Institute Incorporated, 1992). The SAS/LAB Software performs a Levene's test for equal variance, and when there was statistical evidence (based on performing a one-way ANOVA on the absolute deviations of the observations from their respective group means) of unequal variances additional data transformations were performed and outliers removed. Outliers were detected by comparing the studentized residuals to a critical value from a $t$-distribution selected using a Bonferroni-type adjustment. The adjustment is based on the number of observations, $n$, so that the overall probability of a type I error is at most 5 percent. The critical value, $c v$, is given by the following equation:

$$
c v=t\left(d \int_{\text {Error, }} .05 /(2 \times n)\right)
$$

Replicates also were removed from statistical consideration if there were known errors in the pipetting of the gametes to the vials.

A second criterion also was used to compare test means to negative control means. Detectable significance criteria (DSC) have been developed to determine the 95-percent confidence limit based on power analysis of similar tests performed by the USGS lab (Carr and Biedenbach, 1999). At $\alpha=0.01$, the DSC value is 19 percent. The DSC is applied by multiplying the DSC percentage by the reference mean, and subtracting that value from the reference mean to obtain a cutoff value. Results below this value are considered toxic. For instance, the sea urchin fertilization DSC cut-off value at $\alpha=$ 0.01 for a control mean of 95 percent would be 76.95 percent 
[(95-(0.19x95)]. The DSC was developed using the sea urchin Arbacia punctulata, but was used to evaluate these data to aid in comparison to previous studies. A DSC value has not been determined for S. purpuratus.

\section{Results}

\section{Pore-Water Quality Measurements}

Water-quality measurements were conducted on sediment pore water from 30 stations plus the controls. Values for all the water-quality measurements conducted are listed in table 1 (at the back of the report). One sample (\#22) required salinity adjustment before testing. Salinities in test samples ranged from 25 to $31 \%$. Initial dissolved oxygen was greater than 80 percent in all the samples. Total ammonia ranged from 0.12 to 6.99 milligrams per liter $(\mathrm{mg} / \mathrm{L})$, whereas, the unionized ammonia (the most toxic fraction) ranged from 4.2 to 161.6 micrograms per liter $(\mu \mathrm{g} / \mathrm{L})$. No samples exceeded the reported No Observable Effects Concentration (NOEC) for un-ionized ammonia $(170 \mu \mathrm{g} / \mathrm{L})$ for this species (Bailey and others, 1995). Sulfide concentrations were measured in nearly all pore water samples and ranged from 0.01 to $0.019 \mathrm{mg} / \mathrm{L}$.

\section{Sea Urchin Toxicity Testing}

On 21 January 2011, 2 tests were conducted with 15 of the 30 samples in each test, plus the references and controls. The samples were divided into two separate tests to minimize sperm degradation from extended pipetting times. A dilution of eggs collected from a single female was subdivided into two aliquots and used in both tests so that animal variability would not be a factor between the two tests. Sperm from a single male also was used in both tests at the same dilution. The sperm dilutions were made just before pipetting into the samples. Pretest results indicated an optimum sperm dilution of 1 part sperm to 5,000 parts dilution water. Raw data and means from the fertilization tests are given in tables 2 and 3 (at the back of the report). All experimental variables (temperature, lighting, timing, gametes used) remained the same for both tests.

There was one statistical outlying data point in the first test (sample 299, 100-percent dilution, rep 4) (Statistical Analysis Software (SAS) Institute Incorporated, 1992). Four reps in the second test were eliminated from the analysis because of errors in the pipetting of the gametes into those vials ( $\# 53$, 100-percent dilution, rep 2; \#60, 100-percent dilution, rep 5; \#61, 50-percent dilution, rep 4; and \#61, 25-percent dilution, rep 4). The $\mathrm{EC}_{50}$ value for the SDS positive controls were calculated as 2.36 and $2.33 \mathrm{mg} / \mathrm{L}$ for tests 1 and 2, respectively. These results indicate that the tests were more sensitive than previous tests run but were within the 95-percent confidence limits of the historical results for the USGS Corpus Christi, Texas, laboratory for this species of 2.22 to $4.21 \mathrm{mg} / \mathrm{L}$ (appendix 8). Negative control values and dilution water values also were within historical results generated by the USGS Corpus Christi, Texas, laboratory (appendix 9).

In test 1, three samples from stations 32, 85 and 227 indicated significant differences from the reference pore water at the 100-percent pore-water concentration and met the DSC (table 2, fig. 1). Because of minimal standard deviation of the reference, several other samples (\#20, 21, 31, 34, 213, 277 and 299) were significant from the reference at the 100-percent pore-water concentration, but these samples did not meet the detectable significance criteria. None of the diluted pore-water samples produced significantly different results compared to the reference. In test 2, five samples ( $\# 53,59,195,379$ and 42113) indicated significant differences from the negative control pore water at the 100-percent concentration and met the DSC value (table 3, fig. 1). Sample \#59 just barely met the DSC value and, therefore, is only significant at an $\alpha$ of 0.05 . As in the first test, five additional samples (\# 23, 24, 25,26 and 27) were significantly different at the 100-percent pore-water concentration, but did not meet the detectable significance criteria. In test 2 , as in test 1 , none of the diluted pore-water samples produced results that were significantly different than the reference. 


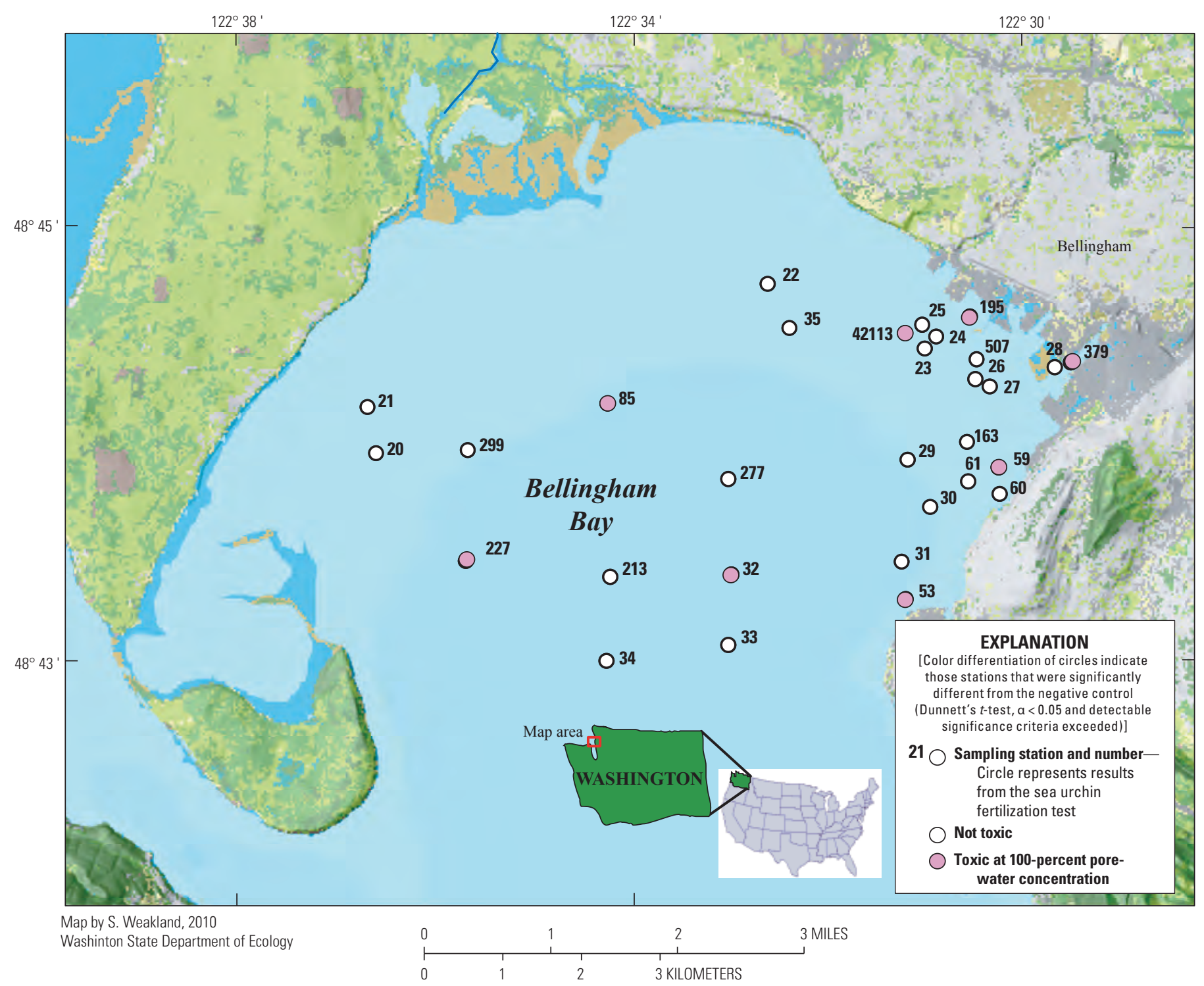

Figure 1. Station locations of sediments and toxicity testing results for the 2010 Urban Waters study in Bellingham Bay, Washington. 


\section{References Cited}

Bailey, H.C., Miller, J.L., Miller, M.J., and Dhaliwal, B.S., 1995, Application of toxicity identification procedures to the echinoderm fertilization assay to identify toxicity in a municipal effluent: Environmental Toxicolology and Chemistry, v. 12 , p. 2,181-2,186.

Bowers, C.E., and Bidwell, J.P., 1978, Ionization of ammonia in seawater; effects of temperature, $\mathrm{pH}$, and salinity: Journal of the Fisheries Research Board of Canada, v. 35, p. $1,012-1,016$.

Carr, R.S., and Biedenbach, J.M.,1999, Use of power analysis to develop detectable significance criteria for sea urchin toxicity tests: Aquatic Ecosystems Health Management, v. 2, p. 413-418.

Carr, R.S. and Chapman, D.C., 1992, Comparison of solidphase and pore-water approaches for assessing the quality of marine and estuarine sediments: Journal of Chemical Ecology, v. 7, p. 19-30.

Carr, R.S. and Chapman, D.C.,1995, Comparison of methods for conducting marine and estuarine sediment pore-water toxicity tests-extraction, storage, and handling techniques: Archives of Environmental Contamination and Toxicology, v. 28 , p. $69-77$.

Carr, R.S., Nipper, M., Field, M. and Biedenbach, J.M., 2006, Coastal circulation and sediment dynamics in Hanalei Bay, Kaua'i, part III, studies of sediment toxicity: U.S. Geological Survey Open-File Report, 2006-1147, 63 p.

Carr, R.S., and Nipper, M., 2008, Measurement of toxicity in reef sediments, in Field, M.E., Cochran, S.A., Logan, J.B., and Storlazzi, C.D., eds., The coral reef of south Moloka'i, Hawai' $i$-Portrait of a sediment-threatened fringe reef: U.S. Geological Survey Scientific Investigations Report 20075101, $190 \mathrm{p}$.

Carr, R.S., Long, E.R., Windom, H.L., Chapman, D.C., Thursby, G., Slone, G.M., and Wolfe, D.A., 1996a, Sediment Quality Assessment Studies of Tampa Bay, Florida: Environmental Toxicology and Chemistry, v. 15, p. 1,2181,231 .

Carr, R.S., Chapman, D.C., Howard, C.L., and Biedenbach, J.M.,1996b, Sediment quality triad assessment survey of the Galveston Bay, Texas system: Ecotoxicology, v. 5, p. 341-364.
Carr, R.S., Chapman, D.C., Presley, B.J., Biedenbach, J.M., Robertson, L., Booth, P., Kilada, R., Wade, T., and Montagna, P.,1997, Sediment pore-water toxicity assessment studies in the vicinity of offshore oil and gas production platforms in the Gulf of Mexico: Canadian Journal of Fisheries and Aquatic Sciences, v. 53, p. 2,618-2,628.

Carr, R.S., Biedenbach, J.M., and Hooten, R., 2001, Sediment quality assessment survey and toxicity identification evaluation studies in Lavaca Bay, Texas, a marine Superfund site: Environmental Toxicology, v. 16, p. 20-30.

Chapman, P.M., 1990, The sediment quality triad approach to determining pollution-induced degradation: Science of the Total Environment, v. 97-98, p. 815-825.

Hamilton, M.A., Russo, R.C., and Thurston, R.V., 1977, Trimmed Spearman-Karber method for estimating median lethal concentrations in toxicity bioassays: Environmental Science Technology, v. 11, p. 714-719; Correction, v. 12, p. 417 (1978).

Long, E., Dutch, M., Aasen, S., Welch, K., and Hameedi, M.J., 2003, Chemical contamination, acute toxicity in laboratory tests, and benthic impacts in sediments of Puget Sound; A summary of results of the joint 1997-1999 Ecology/ National Oceanic and Atmospheric Administration survey: National Oceanic and Atmospheric Administration, Silver Spring, Md., Technical Memo No. 163, 101 p., 1 appendix.

Long, E., Aasen, S., Dutch, M., Welch, K., and Partridge, V., 2008, Sediment quality assessment of the bays and inlets of the San Juan Islands, eastern Strait of Juan de Fuca, and Admiralty Inlet, 2002-2003: Washinton State Department of Ecology, Olympia, Washington, Publication No. 08-03030, 142 p., 7 appendixes.

Morgan, B.J.T., 1992, Analysis of Quantal Response Data: London, England, Chapman and Hall, 511 p.

Statistical Analysis Software (SAS) Institute Incorporated, 1989, SAS/STAT ${ }^{\circledR}$ User's Guide, Version 6, Fourth Edition, Volume 2: Cary, N.C., SAS Institute Inc., 846 p.

Statistical Analysis Software (SAS) Institute Incorporated,1992, SAS/LAB ${ }^{\circledR}$ Software: User's Guide, Version 6, First Edition: Cary, N.C., SAS Institute Inc., 291 p.

Wilson, S., and Partridge, V. 2007, Condition of the outer coastal estuaries of Washington State, 1999, A statistical summary: Washington State Department of Ecology, Olympia, Washington, Publication No. 07-03-012, 76 p., 5 appendixes. 

Tables 1-3 
Table 1. Water-quality measurements after salinity adjustment and original salinity of sediment pore-water samples collected for the 2010 Urban Waters study in Bellingham Bay, Washington.

[Station, sample designation; \%o, parts per thousand; DO, dissolved oxygen; mg/L, milligrams per liter; TAN, total ammonia as nitrogen; UAN, unionized ammonia as nitrogen; $\mu \mathrm{g} / \mathrm{L}$, micrograms per liter; OUS, original unadjusted sample; MFS, Millipore ${ }^{\circledR}$ filtered seawater; $<$, less than; \pm , plus or minus]

\begin{tabular}{|c|c|c|c|c|c|c|c|c|}
\hline Station & $\begin{array}{c}\text { Salinity }^{1} \\
(\% 0)\end{array}$ & $\begin{array}{c}\mathrm{DO}^{2} \\
(\mathrm{mg} / \mathrm{L})\end{array}$ & $\begin{array}{c}\text { Percent } \\
\text { DO }^{3}\end{array}$ & $\mathrm{pH}$ & $\begin{array}{c}\text { TAN }^{4} \\
(\mathrm{mg} / \mathrm{L})\end{array}$ & $\begin{array}{l}\text { UAN }^{5} \\
(\mu \mathrm{g} / \mathrm{L})\end{array}$ & $\begin{array}{c}\text { Sulfide }^{6} \\
\text { (mg/L) }\end{array}$ & $\begin{array}{c}\text { Percent } \\
\text { OUS }^{7}\end{array}$ \\
\hline TXREF $^{8}$ & 30 & 7.43 & 97.7 & 7.90 & 0.51 & 12.6 & 0.010 & 100.0 \\
\hline $\mathrm{MFS}^{9}$ & 31 & 6.69 & 86.5 & 8.05 & .12 & 4.2 & $<.010$ & 100.0 \\
\hline Brine blank $^{10}$ & 30 & 6.70 & 87.6 & 8.01 & .44 & 14.2 & .010 & 100.0 \\
\hline 20 & 30 & 7.19 & 93.4 & 7.27 & 4.33 & 25.7 & .012 & 100.0 \\
\hline 21 & 29 & 7.19 & 93.0 & 7.57 & 4.06 & 48.5 & .015 & 100.0 \\
\hline 22 & 25 & 7.56 & 97.6 & 7.68 & 1.11 & 16.9 & .013 & 93.8 \\
\hline 23 & 30 & 6.89 & 88.7 & 7.70 & 4.93 & 77.8 & .015 & 100.0 \\
\hline 24 & 29 & 6.97 & 89.6 & 7.15 & 3.03 & 13.8 & .015 & 100.0 \\
\hline 25 & 29 & 7.00 & 90.2 & 7.22 & 3.02 & 15.9 & .016 & 100.0 \\
\hline 26 & 30 & 7.19 & 92.5 & 7.25 & 3.72 & 21.3 & .015 & 100.0 \\
\hline 27 & 29 & 7.26 & 93.7 & 7.62 & 3.87 & 51.3 & .016 & 100.0 \\
\hline 28 & 29 & 7.18 & 92.9 & 7.54 & 4.62 & 51.7 & .015 & 100.0 \\
\hline 29 & 30 & 7.09 & 91.7 & 7.86 & 6.99 & 161.2 & .016 & 100.0 \\
\hline 30 & 30 & 6.81 & 89.1 & 7.59 & 4.68 & 58.7 & .015 & 100.0 \\
\hline 31 & 30 & 6.97 & 90.3 & 7.32 & 2.76 & 18.7 & .016 & 100.0 \\
\hline 32 & 30 & 7.10 & 92.0 & 7.19 & 4.64 & 23.4 & .018 & 100.0 \\
\hline 33 & 31 & 7.12 & 92.2 & 7.48 & 2.45 & 23.7 & .015 & 100.0 \\
\hline 34 & 30 & 7.42 & 96.6 & 7.37 & 3.77 & 28.6 & .016 & 100.0 \\
\hline 35 & 30 & 7.39 & 96.0 & 7.76 & 5.22 & 96.1 & .016 & 100.0 \\
\hline 53 & 30 & 7.15 & 93.2 & 7.70 & 6.80 & 109.3 & .016 & 100.0 \\
\hline 59 & 30 & 7.23 & 93.9 & 7.15 & 4.48 & 20.3 & .016 & 100.0 \\
\hline 60 & 30 & 7.36 & 95.5 & 7.30 & 4.25 & 27.1 & .015 & 100.0 \\
\hline 61 & 30 & 7.11 & 92.2 & 7.39 & 4.11 & 32.5 & .015 & 100.0 \\
\hline 85 & 30 & 7.14 & 92.5 & 7.47 & 5.30 & 50.6 & .016 & 100.0 \\
\hline 163 & 30 & 7.40 & 96.0 & 7.30 & 3.86 & 24.9 & .015 & 100.0 \\
\hline 195 & 29.5 & 7.25 & 94.1 & 7.85 & 5.93 & 131.9 & .017 & 100.0 \\
\hline 213 & 30.5 & 7.39 & 95.9 & 7.57 & 2.56 & 30.6 & .015 & 100.0 \\
\hline 227 & 30 & 7.25 & 94.1 & 7.46 & 4.46 & 41.3 & .019 & 100.0 \\
\hline 277 & 30 & 7.48 & 97.2 & 7.50 & 2.44 & 24.5 & .015 & 100.0 \\
\hline 299 & 30 & 7.32 & 95.0 & 7.71 & 5.77 & 93.3 & .016 & 100.0 \\
\hline 379 & 30 & 7.29 & 94.9 & 7.67 & 4.18 & 62.3 & .016 & 100.0 \\
\hline 507 & 29 & 7.31 & 95.1 & 7.30 & 3.53 & 22.4 & .016 & 100.0 \\
\hline 42113 & 29 & 6.95 & 90.4 & 7.16 & 3.31 & 15.6 & .016 & 100.0 \\
\hline
\end{tabular}

${ }^{1}$ Salinity of sample before adjustment. Sample adjusted to $30 \pm 1 \%$.

${ }^{2}$ Dissolved oxygen.

${ }^{3}$ Percent saturation of dissolved oxygen.

${ }^{4}$ Total ammonia as nitrogen.

${ }^{5}$ Un-ionized ammonia as nitrogen.

${ }^{6}$ Measured as $\mathrm{S}^{-2}$.

${ }^{7}$ Percent of original sample after salinity adjustment.

${ }^{8}$ Negative control pore water extracted from sediment collected in Aransas Bay, Texas.

${ }^{9}$ Millipore ${ }^{\circledR}$ filtered seawater diluent.

${ }^{10}$ Blank consisting of TXREF diluted to $25 \%$ with Milli-Q ${ }^{\circledR}$ purified water and then brought back to $30 \%$ with hypersaline brine. 
Table 2. Sea urchin fertilization test raw data and means for sediment pore-water samples in test one of the Urban Waters 2010 study in Bellingham Bay, Washington.

[Station, sample designation; WQAS, water quality adjusted sample; Rep, replicate; SD, standard deviation; Sig., Significance; nc, negative control; ++ , statistical difference at alpha less than or equal to 0.01 ; ns, not significant; $* *$, statistical difference and detectable significance criteria exceedance at alpha less than or equal to 0.01 ; MFS, Millipore ${ }^{\circledR}$ filtered seawater; SDS, sodium dodecyl sulfate; $\mathrm{mg} / \mathrm{L}$, milligrams per liter; \%, parts per thousand]

\begin{tabular}{|c|c|c|c|c|c|c|c|c|c|c|}
\hline \multirow{2}{*}{ Station } & \multirow{2}{*}{$\begin{array}{l}\text { Percent } \\
\text { WOAS }^{1}\end{array}$} & \multicolumn{5}{|c|}{ Percent fertilized } & \multirow{2}{*}{ Mean } & \multirow{2}{*}{ SD } & \multirow{2}{*}{ Sig. ${ }^{2}$} & \multirow{2}{*}{$\begin{array}{l}\text { Percent of } \\
\text { control }^{3}\end{array}$} \\
\hline & & Rep 1 & Rep 2 & Rep 3 & Rep 4 & $\operatorname{Rep} 5$ & & & & \\
\hline \multirow[t]{6}{*}{$\mathrm{TXREF}^{4}$} & 100 & 98 & 99 & 100 & 98 & 98 & 98.3 & 1.06 & $\mathrm{nc}$ & 100.0 \\
\hline & & 100 & 97 & 97 & 98 & 98 & & & & \\
\hline & 50 & 99 & 99 & 100 & 100 & 100 & 99.0 & .94 & $\mathrm{nc}$ & 100.0 \\
\hline & & 99 & 99 & 97 & 99 & 98 & & & & \\
\hline & 25 & 95 & 100 & 100 & 99 & 99 & 98.6 & 1.78 & $\mathrm{nc}$ & 100.0 \\
\hline & & 99 & 100 & 96 & 98 & 100 & & & & \\
\hline \multirow[t]{3}{*}{20} & 100 & 92 & 92 & 89 & 92 & 94 & 91.8 & 1.79 & ++ & 93.4 \\
\hline & 50 & 98 & 100 & 100 & 98 & 98 & 98.8 & 1.10 & ns & 99.8 \\
\hline & 25 & 99 & 100 & 99 & 100 & 98 & 99.2 & .84 & ns & 100.6 \\
\hline \multirow[t]{3}{*}{21} & 100 & 96 & 91 & 89 & 94 & 85 & 91.0 & 4.30 & ++ & 92.6 \\
\hline & 50 & 99 & 100 & 99 & 100 & 99 & 99.4 & .55 & ns & 100.4 \\
\hline & 25 & 99 & 100 & 98 & 98 & 98 & 98.6 & .89 & ns & 100.0 \\
\hline \multirow[t]{3}{*}{22} & 100 & 97 & 100 & 100 & 99 & 100 & 99.2 & 1.30 & $\mathrm{~ns}$ & 100.9 \\
\hline & 50 & 100 & 99 & 99 & 100 & 99 & 99.4 & .55 & $\mathrm{~ns}$ & 100.4 \\
\hline & 25 & 100 & 100 & 100 & 100 & 99 & 99.8 & .45 & ns & 101.2 \\
\hline \multirow[t]{3}{*}{29} & 100 & 95 & 98 & 99 & 97 & 96 & 97.0 & 1.58 & ns & 98.7 \\
\hline & 50 & 99 & 100 & 99 & 100 & 99 & 99.4 & .55 & $\mathrm{~ns}$ & 100.4 \\
\hline & 25 & 99 & 98 & 100 & 99 & 98 & 98.8 & .84 & ns & 100.2 \\
\hline \multirow[t]{3}{*}{30} & 100 & 98 & 97 & 96 & 98 & 97 & 97.2 & .84 & $\mathrm{~ns}$ & 98.9 \\
\hline & 50 & 98 & 100 & 100 & 98 & 100 & 99.2 & 1.10 & ns & 100.2 \\
\hline & 25 & 97 & 99 & 100 & 100 & 96 & 98.0 & 1.83 & ns & 99.4 \\
\hline \multirow[t]{3}{*}{31} & 100 & 84 & 77 & 88 & 86 & 87 & 84.4 & 4.39 & ++ & 85.9 \\
\hline & 50 & 100 & 98 & 95 & 100 & 99 & 98.4 & 2.07 & $\mathrm{~ns}$ & 99.4 \\
\hline & 25 & 98 & 100 & 98 & 100 & 97 & 98.6 & 1.34 & $\mathrm{~ns}$ & 100.0 \\
\hline \multirow[t]{3}{*}{32} & 100 & 45 & 41 & 49 & 39 & 39 & 42.6 & 4.34 & $* *$ & 43.3 \\
\hline & 50 & 96 & 100 & 97 & 98 & 98 & 97.8 & 1.48 & ns & 98.8 \\
\hline & 25 & 99 & 97 & 99 & 98 & 99 & 98.4 & .89 & $\mathrm{~ns}$ & 99.8 \\
\hline \multirow[t]{3}{*}{33} & 100 & 97 & 96 & 98 & 97 & 99 & 97.5 & 1.29 & $\mathrm{~ns}$ & 99.2 \\
\hline & 50 & 99 & 96 & 96 & 99 & 98 & 97.6 & 1.52 & $\mathrm{~ns}$ & 98.6 \\
\hline & 25 & 98 & 98 & 99 & 99 & 99 & 98.6 & .55 & ns & 100.0 \\
\hline \multirow[t]{3}{*}{34} & 100 & 86 & 85 & 89 & 90 & 88 & 87.6 & 2.07 & ++ & 89.1 \\
\hline & 50 & 99 & 98 & 97 & 100 & 100 & 98.8 & 1.30 & $\mathrm{~ns}$ & 99.8 \\
\hline & 25 & 100 & 99 & 99 & 95 & 100 & 98.6 & 2.07 & ns & 100.0 \\
\hline \multirow[t]{3}{*}{35} & 100 & 98 & 96 & 98 & 97 & 97 & 97.2 & .84 & $\mathrm{~ns}$ & 98.9 \\
\hline & 50 & 96 & 100 & 98 & 98 & 99 & 98.2 & 1.48 & $\mathrm{~ns}$ & 99.2 \\
\hline & 25 & 99 & 100 & 99 & 96 & 99 & 98.6 & 1.52 & ns & 100.0 \\
\hline \multirow[t]{3}{*}{85} & 100 & 68 & 84 & 48 & 66 & 68 & 66.8 & 12.77 & $* *$ & 68.0 \\
\hline & 50 & 100 & 99 & 100 & 99 & 98 & 99.2 & .84 & ns & 100.2 \\
\hline & 25 & 99 & 98 & 100 & 100 & 100 & 99.4 & .89 & $\mathrm{~ns}$ & 100.8 \\
\hline
\end{tabular}


Table 2. Sea urchin fertilization test raw data and means for sediment pore-water samples in test one of the Urban Waters 2010 study in Bellingham Bay, Washington.-Continued

[Station, sample designation; WQAS, water quality adjusted sample; Rep, replicate; SD, standard deviation; Sig., Significance; nc, negative control; ++ , statistical difference at alpha less than or equal to 0.01 ; ns, not significant; **, statistical difference and detectable significance criteria exceedance at alpha less than or equal to 0.01 ; MFS, Millipore ${ }^{\circledR}$ filtered seawater; SDS, sodium dodecyl sulfate; $\mathrm{mg} / \mathrm{L}$, milligrams per liter; \%o, parts per thousand]

\begin{tabular}{|c|c|c|c|c|c|c|c|c|c|c|}
\hline \multirow{2}{*}{ Station } & \multirow{2}{*}{$\begin{array}{l}\text { Percent } \\
\text { WOAS }^{1}\end{array}$} & \multicolumn{5}{|c|}{ Percent fertilized } & \multirow{2}{*}{ Mean } & \multirow{2}{*}{ SD } & \multirow{2}{*}{ Sig. ${ }^{2}$} & \multirow{2}{*}{$\begin{array}{l}\text { Percent of } \\
\text { control }^{3}\end{array}$} \\
\hline & & Rep 1 & Rep 2 & Rep 3 & Rep 4 & Rep 5 & & & & \\
\hline \multirow[t]{3}{*}{213} & 100 & 95 & 88 & 89 & 85 & 81 & 87.6 & 5.18 & ++ & 89.1 \\
\hline & 50 & 99 & 100 & 95 & 98 & 99 & 98.2 & 1.92 & $\mathrm{~ns}$ & 99.2 \\
\hline & 25 & 100 & 96 & 99 & 99 & 100 & 98.8 & 1.64 & $\mathrm{~ns}$ & 100.2 \\
\hline \multirow[t]{3}{*}{227} & 100 & 8 & 6 & 2 & 9 & 2 & 5.4 & 3.29 & $* *$ & 5.5 \\
\hline & 50 & 96 & 94 & 99 & 99 & 94 & 96.4 & 2.51 & $\mathrm{~ns}$ & 97.4 \\
\hline & 25 & 99 & 98 & 96 & 98 & 97 & 97.6 & 1.14 & $\mathrm{~ns}$ & 99.0 \\
\hline \multirow[t]{3}{*}{277} & 100 & 85 & 90 & 87 & 88 & 85 & 87.0 & 2.12 & ++ & 88.5 \\
\hline & 50 & 100 & 98 & 97 & 98 & 96 & 97.8 & 1.48 & $\mathrm{~ns}$ & 98.8 \\
\hline & 25 & 98 & 100 & 98 & 100 & 99 & 99.0 & 1.00 & $\mathrm{~ns}$ & 100.4 \\
\hline \multirow[t]{3}{*}{299} & 100 & 87 & 86 & 84 & ${ }^{5} 60$ & 83 & 85.0 & 1.83 & ++ & 86.5 \\
\hline & 50 & 100 & 99 & 99 & 100 & 99 & 99.4 & .55 & $\mathrm{~ns}$ & 100.4 \\
\hline & 25 & 100 & 100 & 100 & 98 & 100 & 99.6 & .89 & ns & 101.0 \\
\hline \multirow[t]{2}{*}{$\mathrm{MFS}^{6}$} & 100 & 100 & 98 & 100 & 98 & 99 & 98.4 & 1.26 & ns & 100.1 \\
\hline & & 97 & 100 & 97 & 97 & 98 & & & & \\
\hline Brine blank ${ }^{7}$ & 100 & 96 & 99 & 100 & 100 & 100 & 99.0 & 1.73 & ns & 100.7 \\
\hline \multirow[t]{4}{*}{$\mathrm{SDS}^{8}$} & 10 & 0 & 0 & 0 & 0 & 0 & 0.0 & 0.00 & ns & 0.0 \\
\hline & 5 & 0 & 0 & 0 & 0 & 0 & 0.0 & 0.00 & $\mathrm{~ns}$ & 0.0 \\
\hline & 2.5 & 37 & 41 & 46 & 49 & 48 & 44.2 & 5.07 & $* *$ & 45.0 \\
\hline & 1.25 & 93 & 90 & 88 & 89 & 91 & 90.2 & 1.92 & ++ & 91.8 \\
\hline
\end{tabular}

${ }^{1}$ Percent of water quality adjusted pore-water sampled or concentration in $\mathrm{mg} / \mathrm{L}$ for SDS positive control.

${ }^{2}$ Significant difference determined using Dunnett's $t$-test and detectable significance criteria.

${ }^{3}$ Percent of TXREF negative control.

${ }^{4}$ Negative control pore water extracted from sediment collected in Aransas Bay, Texas.

${ }^{5}$ Statistical outlier removed from analysis.

${ }^{6}$ Millipore ${ }^{\circledR}$ filtered seawater diluent.

${ }^{7}$ Blank consisting of TXREF diluted to $25 \%$ with Milli- $Q^{\circledR}$ purified water and then brought back to $30 \%$ with hypersaline brine.

${ }^{8}$ Sodium dodecyl sulfate positive control (in $\mathrm{mg} / \mathrm{L}$ ). 
Table 3. Sea urchin fertilization test raw data and means for sediment pore-water samples in test two of the Urban Waters 2010 study in Bellingham Bay, Washington.

[Station, sample designation; WQAS, water quality adjusted sample; Rep, replicate; SD, standard deviation; Sig., Significance; nc, negative control; ++ , statistical difference at alpha less than or equal to 0.01 ; ns, not significant; ${ }^{* *}$, statistical difference and detectable significance criteria exceedance at alpha less than or equal to 0.01 ; ${ }^{*}$, statistical difference and detectable significance criteria exceedance at alpha less than or equal to 0.05 ; MFS, Millipore ${ }^{\circledR}$ filtered seawater; SDS, sodium dodecyl sulfate; $\mathrm{mg} / \mathrm{L}$, milligrams per liter; \%o, parts per thousand]

\begin{tabular}{|c|c|c|c|c|c|c|c|c|c|c|}
\hline \multirow{2}{*}{ Station } & \multirow{2}{*}{$\begin{array}{l}\text { Percent } \\
\text { WOAS }^{1}\end{array}$} & \multicolumn{5}{|c|}{ Percent fertilized } & \multirow{2}{*}{ Mean } & \multirow{2}{*}{ SD } & \multirow{2}{*}{ Sig. ${ }^{2}$} & \multirow{2}{*}{$\begin{array}{c}\text { Percent o } \\
\text { control }^{3}\end{array}$} \\
\hline & & Rep 1 & Rep 2 & Rep 3 & Rep 4 & Rep 5 & & & & \\
\hline \multirow[t]{6}{*}{$\mathrm{TXREF}^{4}$} & 100 & 98 & 99 & 100 & 99 & 99 & 98.5 & 1.18 & $\mathrm{nc}$ & 100.0 \\
\hline & & 97 & 97 & 100 & 99 & 97 & & & & \\
\hline & 50 & 98 & 98 & 99 & 99 & 100 & 98.8 & .79 & nc & 100.0 \\
\hline & & 98 & 99 & 98 & 100 & 99 & & & & \\
\hline & 25 & 99 & 100 & 98 & 100 & 100 & 99.5 & .71 & nc & 100.0 \\
\hline & & 99 & 100 & 99 & 100 & 100 & & & & \\
\hline \multirow[t]{3}{*}{23} & 100 & 94 & 98 & 97 & 96 & 92 & 95.4 & 2.41 & ++ & 96.9 \\
\hline & 50 & 99 & 99 & 99 & 100 & 100 & 99.4 & .55 & ns & 100.6 \\
\hline & 25 & 100 & 100 & 100 & 100 & 99 & 99.8 & .45 & ns & 100.3 \\
\hline \multirow[t]{3}{*}{24} & 100 & 92 & 95 & 95 & 91 & 90 & 92.6 & 2.30 & ++ & 94.0 \\
\hline & 50 & 100 & 98 & 99 & 100 & 100 & 99.4 & .89 & ns & 100.6 \\
\hline & 25 & 99 & 99 & 100 & 100 & 99 & 99.4 & .55 & ns & 99.9 \\
\hline \multirow[t]{3}{*}{25} & 100 & 94 & 85 & 85 & 86 & 80 & 86.0 & 5.05 & ++ & 87.3 \\
\hline & 50 & 96 & 98 & 98 & 100 & 100 & 98.4 & 1.67 & ns & 99.6 \\
\hline & 25 & 99 & 100 & 100 & 99 & 99 & 99.4 & .55 & ns & 99.9 \\
\hline \multirow[t]{3}{*}{26} & 100 & 87 & 92 & 94 & 92 & 89 & 90.8 & 2.77 & ++ & 92.2 \\
\hline & 50 & 97 & 99 & 96 & 99 & 99 & 98.0 & 1.41 & ns & 99.2 \\
\hline & 25 & 98 & 95 & 98 & 100 & 99 & 98.0 & 1.87 & ns & 98.5 \\
\hline \multirow[t]{3}{*}{27} & 100 & 83 & 88 & 90 & 83 & 91 & 87.0 & 3.81 & ++ & 88.3 \\
\hline & 50 & 98 & 99 & 100 & 98 & 98 & 98.6 & .89 & ns & 99.8 \\
\hline & 25 & 98 & 100 & 100 & 100 & 99 & 99.4 & .89 & ns & 99.9 \\
\hline \multirow[t]{3}{*}{28} & 100 & 98 & 100 & 98 & 100 & 98 & 98.8 & 1.10 & ns & 100.3 \\
\hline & 50 & 100 & 100 & 100 & 99 & 99 & 99.6 & .55 & $\mathrm{~ns}$ & 100.8 \\
\hline & 25 & 98 & 99 & 96 & 100 & 100 & 98.6 & 1.67 & ns & 99.1 \\
\hline \multirow[t]{3}{*}{53} & 100 & 66 & ${ }^{5} 0$ & 80 & 72 & 75 & 73.3 & 5.85 & $* *$ & 74.4 \\
\hline & 50 & 100 & 99 & 100 & 98 & 97 & 98.8 & 1.30 & ns & 100.0 \\
\hline & 25 & 99 & 98 & 98 & 100 & 97 & 98.4 & 1.14 & ns & 98.9 \\
\hline \multirow[t]{3}{*}{59} & 100 & 83 & 81 & 80 & 83 & 88 & 83.0 & 3.56 & $*$ & 84.3 \\
\hline & 50 & 99 & 99 & 98 & 99 & 96 & 98.2 & 1.30 & ns & 99.4 \\
\hline & 25 & 100 & 99 & 99 & 98 & 98 & 98.8 & .84 & ns & 99.3 \\
\hline \multirow[t]{3}{*}{60} & 100 & 99 & 99 & 97 & 99 & ${ }^{5} 0$ & 98.5 & 1.00 & ns & 100.0 \\
\hline & 50 & 100 & 98 & 100 & 96 & 99 & 98.6 & 1.67 & ns & 99.8 \\
\hline & 25 & 100 & 98 & 96 & 99 & 95 & 97.6 & 2.07 & $\mathrm{~ns}$ & 98.1 \\
\hline \multirow[t]{3}{*}{61} & 100 & 96 & 98 & 98 & 99 & 98 & 97.8 & 1.10 & ns & 99.3 \\
\hline & 50 & 100 & 96 & 99 & ${ }^{5} 0$ & 99 & 98.5 & 1.73 & ns & 99.7 \\
\hline & 25 & 100 & 96 & 99 & ${ }^{5} 0$ & 99 & 98.5 & 1.73 & ns & 99.0 \\
\hline \multirow[t]{3}{*}{163} & 100 & 93 & 99 & 96 & 99 & 97 & 96.8 & 2.49 & ns & 98.3 \\
\hline & 50 & 100 & 100 & 99 & 99 & 99 & 99.4 & .55 & ns & 100.6 \\
\hline & 25 & 99 & 100 & 99 & 100 & 99 & 99.4 & .55 & ns & 99.9 \\
\hline
\end{tabular}


Table 3. Sea urchin fertilization test raw data and means for sediment pore-water samples in test two of the Urban Waters 2010 study in Bellingham Bay, Washington.-Continued

[Station, sample designation; WQAS, water quality adjusted sample; Rep, replicate; SD, standard deviation; Sig., Significance; nc, negative control; ++ , statistical difference at alpha less than or equal to 0.01 ; ns, not significant; $* *$, statistical difference and detectable significance criteria exceedance at alpha less than or equal to 0.01 ; ${ }^{*}$, statistical difference and detectable significance criteria exceedance at alpha less than or equal to 0.05 ; MFS, Millipore ${ }^{\circledR}$ filtered seawater; SDS, sodium dodecyl sulfate; $\mathrm{mg} / \mathrm{L}$, milligrams per liter; \%o, parts per thousand]

\begin{tabular}{|c|c|c|c|c|c|c|c|c|c|c|}
\hline \multirow{2}{*}{ Station } & \multirow{2}{*}{$\begin{array}{l}\text { Percent } \\
\text { WOAS }^{1}\end{array}$} & \multicolumn{5}{|c|}{ Percent fertilized } & \multirow{2}{*}{ Mean } & \multirow{2}{*}{ SD } & \multirow{2}{*}{ Sig. $^{2}$} & \multirow{2}{*}{$\begin{array}{l}\text { Percent of } \\
\text { control }^{3}\end{array}$} \\
\hline & & Rep 1 & Rep 2 & Rep 3 & Rep 4 & Rep 5 & & & & \\
\hline \multirow[t]{3}{*}{195} & 100 & 74 & 80 & 74 & 76 & 71 & 75.0 & 3.32 & $* *$ & 76.1 \\
\hline & 50 & 98 & 100 & 100 & 100 & 100 & 99.6 & .89 & ns & 100.8 \\
\hline & 25 & 99 & 98 & 100 & 99 & 99 & 99.0 & .71 & ns & 99.5 \\
\hline \multirow[t]{3}{*}{379} & 100 & 72 & 79 & 79 & 70 & 84 & 76.8 & 5.72 & $* *$ & 78.0 \\
\hline & 50 & 100 & 100 & 99 & 100 & 97 & 99.2 & 1.30 & $\mathrm{~ns}$ & 100.4 \\
\hline & 25 & 97 & 97 & 100 & 100 & 99 & 98.6 & 1.52 & ns & 99.1 \\
\hline \multirow[t]{3}{*}{507} & 100 & 93 & 95 & 98 & 98 & 97 & 96.2 & 2.17 & ns & 97.7 \\
\hline & 50 & 100 & 98 & 97 & 98 & 100 & 98.6 & 1.34 & ns & 99.8 \\
\hline & 25 & 100 & 98 & 99 & 99 & 100 & 99.2 & .84 & ns & 99.7 \\
\hline \multirow[t]{3}{*}{42113} & 100 & 42 & 44 & 29 & 25 & 49 & 41.0 & 8.52 & $* *$ & 41.6 \\
\hline & 50 & 100 & 99 & 99 & 98 & 97 & 98.6 & 1.14 & $\mathrm{~ns}$ & 99.8 \\
\hline & 25 & 99 & 99 & 97 & 93 & 99 & 97.4 & 2.61 & ns & 97.9 \\
\hline \multirow[t]{2}{*}{$\mathrm{MFS}^{6}$} & 100 & 99 & 99 & 99 & 99 & 99 & 98.9 & .74 & ns & 100.4 \\
\hline & & 99 & 97 & 99 & 99 & 100 & & & & \\
\hline Brine blank $^{7}$ & 100 & 100 & 100 & 100 & 100 & 99 & 99.8 & .45 & $\mathrm{~ns}$ & 101.3 \\
\hline \multirow[t]{4}{*}{$\mathrm{SDS}^{8}$} & 10 & 0 & 0 & 0 & 0 & 0 & 0.0 & 0.00 & $\mathrm{~ns}$ & 0.0 \\
\hline & 5 & 0 & 0 & 0 & 0 & 0 & 0.0 & 0.00 & ns & 0.0 \\
\hline & 2.5 & 43 & 49 & 46 & 43 & 45 & 45.2 & 2.49 & $* *$ & 45.9 \\
\hline & 1.25 & 97 & 93 & 92 & 95 & 91 & 93.6 & 2.41 & ++ & 95.0 \\
\hline
\end{tabular}

${ }^{1}$ Percent of water quality adjusted pore-water sampled or concentration in $\mathrm{mg} / \mathrm{L}$ for SDS positive control.

${ }^{2}$ Significant difference determined using Dunnett's $t$-test and detectable significance criteria.

${ }^{3}$ Percent of TXREF control.

${ }^{4}$ Negative control pore water extracted from sediment collected in Aransas Bay, Texas.

${ }^{5}$ Statistical outlier removed from analysis.

${ }^{6}$ Millipore ${ }^{\circledR}$ filtered seawater diluent.

${ }^{7}$ Blank consisting of TXREF diluted to $25 \%$ with Milli-Q ${ }^{\circledR}$ purified water and then brought back to $30 \%$ with hypersaline brine.

${ }^{8}$ Sodium dodecyl sulfate positive control (in $\mathrm{mg} / \mathrm{L}$ ). 
Appendixes 1-9 


\section{Appendix 1}

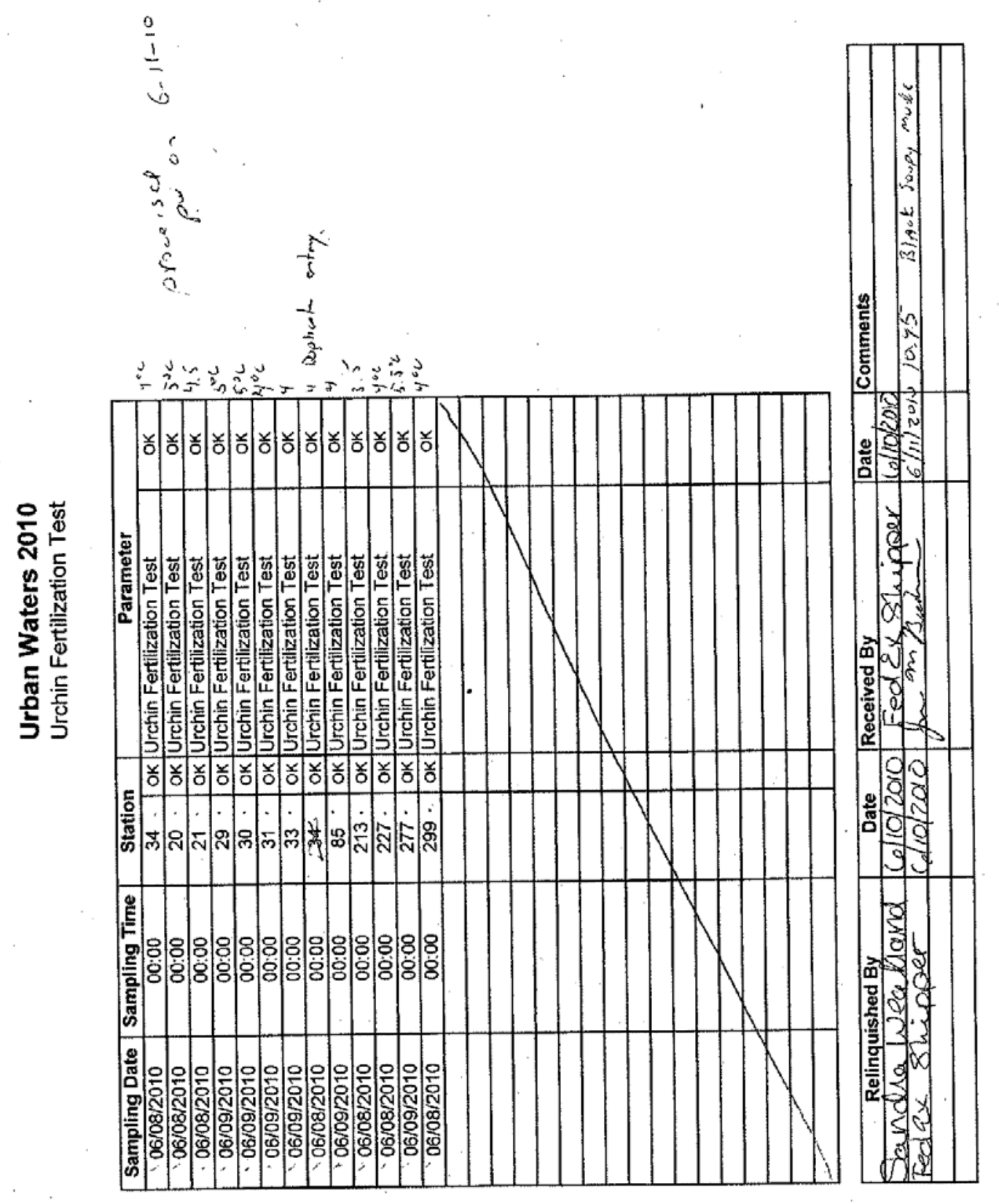

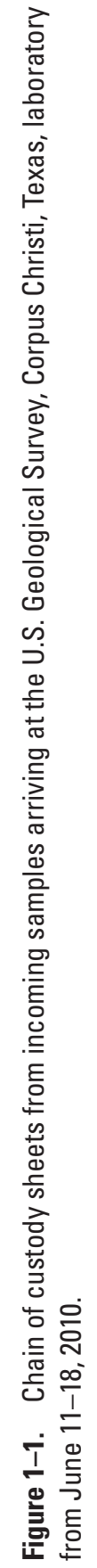


$=\sum_{-\infty}^{2}$

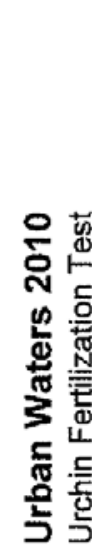

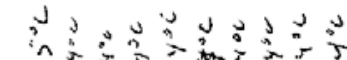
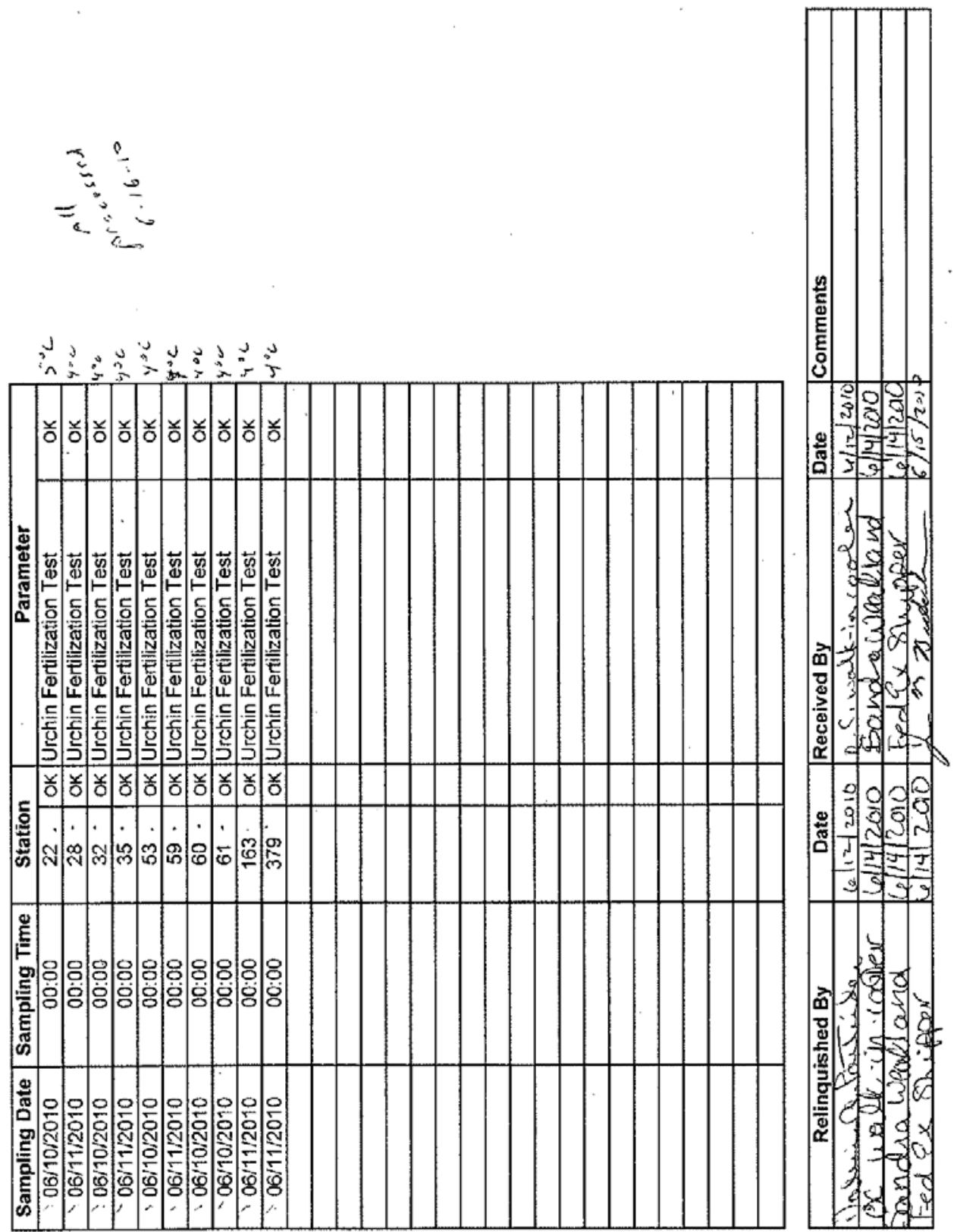

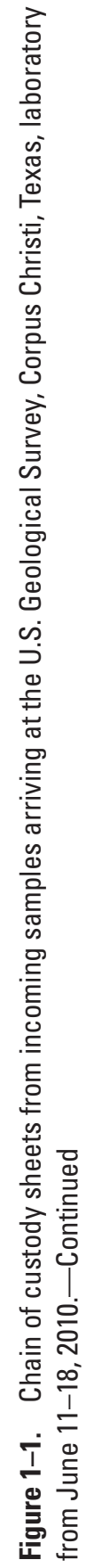


is

$\hat{\jmath}$

$\$$

10

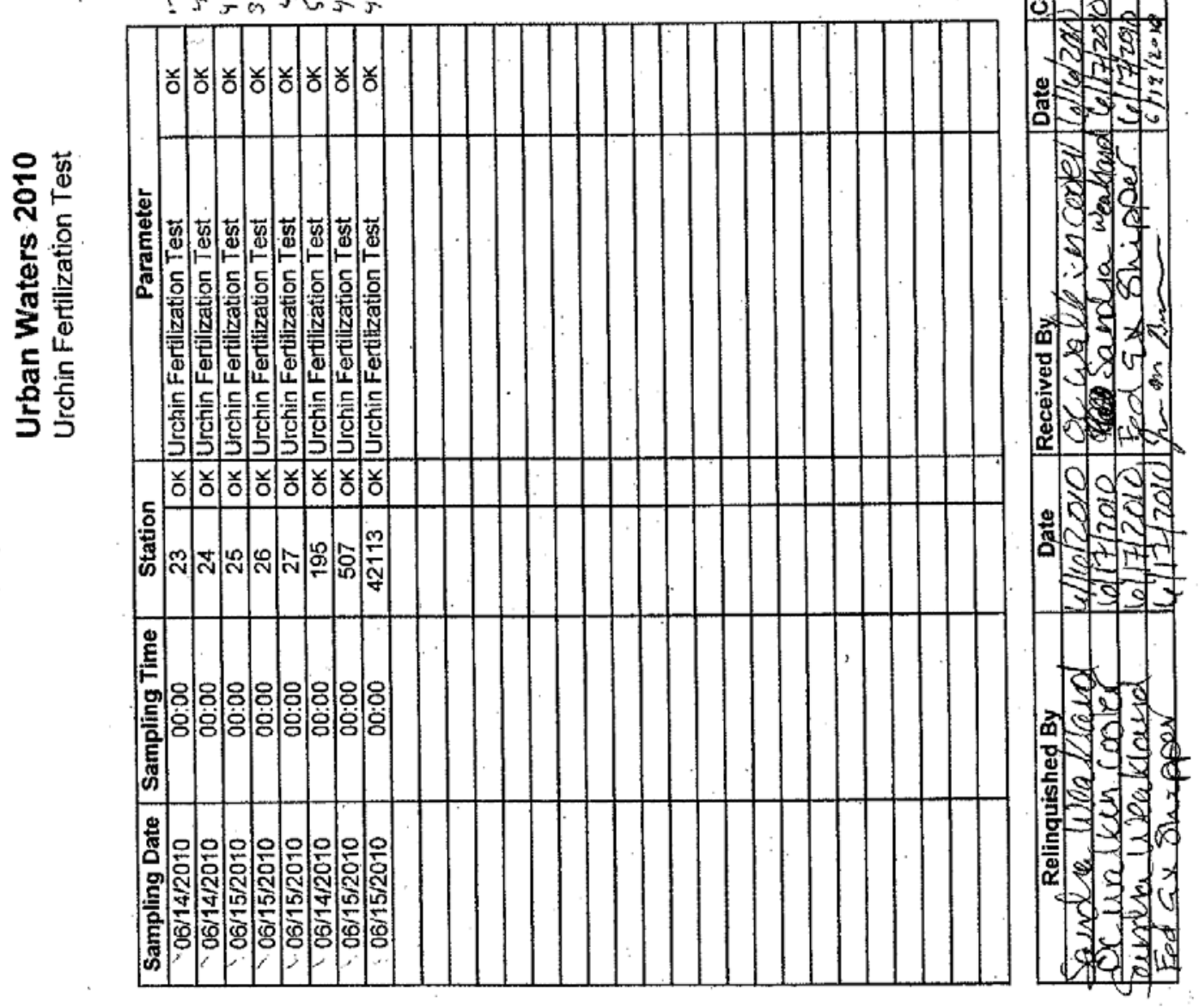

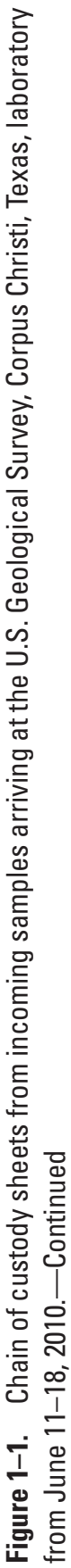


Appendix 2. Table of relevant sample dates, pore-water volumes and holding times for the 2010 Urban Waters study in Belingham Bay, Washington.

[Station, sample designation]

\begin{tabular}{|c|c|c|c|c|c|c|c|}
\hline Station & $\begin{array}{c}\text { Date } \\
\text { collected }^{1}\end{array}$ & $\begin{array}{c}\text { Date } \\
\text { shipped }^{2}\end{array}$ & $\begin{array}{c}\text { Date } \\
\text { received }^{3}\end{array}$ & $\begin{array}{c}\text { Date } \\
\text { extracted }^{4}\end{array}$ & $\begin{array}{c}\text { Volume } \\
\text { extracted }^{5}\end{array}$ & $\begin{array}{l}\text { Holding } \\
\text { time }^{6}\end{array}$ & $\begin{array}{c}\text { Date } \\
\text { tested }^{7}\end{array}$ \\
\hline 20 & $6 / 8 / 2010$ & $6 / 10 / 2010$ & $6 / 11 / 2010$ & $6 / 11 / 2010$ & 450 & 3 & $1 / 21 / 2011$ \\
\hline 21 & 6/8/2010 & $6 / 10 / 2010$ & $6 / 11 / 2010$ & $6 / 11 / 2010$ & 450 & 3 & $1 / 21 / 2011$ \\
\hline 22 & $6 / 10 / 2010$ & $6 / 14 / 2010$ & $6 / 15 / 2010$ & $6 / 16 / 2010$ & 450 & 6 & $1 / 21 / 2011$ \\
\hline 23 & $6 / 14 / 2010$ & $6 / 17 / 2010$ & $6 / 18 / 2010$ & $6 / 18 / 2010$ & 450 & 4 & $1 / 21 / 2011$ \\
\hline 24 & $6 / 14 / 2010$ & $6 / 17 / 2010$ & $6 / 18 / 2010$ & $6 / 18 / 2010$ & 450 & 4 & $1 / 21 / 2011$ \\
\hline 25 & $6 / 15 / 2010$ & $6 / 17 / 2010$ & $6 / 18 / 2010$ & $6 / 18 / 2010$ & 450 & 3 & $1 / 21 / 2011$ \\
\hline 26 & $6 / 15 / 2010$ & $6 / 17 / 2010$ & $6 / 18 / 2010$ & $6 / 18 / 2010$ & 450 & 3 & $1 / 21 / 2011$ \\
\hline 27 & $6 / 15 / 2010$ & $6 / 17 / 2010$ & $6 / 18 / 2010$ & $6 / 18 / 2010$ & 450 & 3 & $1 / 21 / 2011$ \\
\hline 28 & $6 / 11 / 2010$ & $6 / 14 / 2010$ & $6 / 15 / 2010$ & $6 / 16 / 2010$ & 450 & 5 & $1 / 21 / 2011$ \\
\hline 29 & $6 / 9 / 2010$ & $6 / 10 / 2010$ & $6 / 11 / 2010$ & $6 / 11 / 2010$ & 450 & 2 & $1 / 21 / 2011$ \\
\hline 30 & 6/9/2010 & $6 / 10 / 2010$ & $6 / 11 / 2010$ & $6 / 11 / 2010$ & 450 & 2 & $1 / 21 / 2011$ \\
\hline 31 & $6 / 9 / 2010$ & $6 / 10 / 2010$ & $6 / 11 / 2010$ & $6 / 11 / 2010$ & 450 & 2 & $1 / 21 / 2011$ \\
\hline 32 & $6 / 10 / 2010$ & $6 / 14 / 2010$ & $6 / 15 / 2010$ & $6 / 16 / 2010$ & 450 & 6 & $1 / 21 / 2011$ \\
\hline 33 & 6/9/2010 & $6 / 10 / 2010$ & $6 / 11 / 2010$ & $6 / 11 / 2010$ & 450 & 2 & $1 / 21 / 2011$ \\
\hline 34 & $6 / 8 / 2010$ & $6 / 10 / 2010$ & $6 / 11 / 2010$ & $6 / 11 / 2010$ & 450 & 3 & $1 / 21 / 2011$ \\
\hline 35 & $6 / 11 / 2010$ & $6 / 14 / 2010$ & $6 / 15 / 2010$ & $6 / 16 / 2010$ & 450 & 5 & $1 / 21 / 2011$ \\
\hline 53 & $6 / 10 / 2010$ & $6 / 14 / 2010$ & $6 / 15 / 2010$ & $6 / 16 / 2010$ & 450 & 6 & $1 / 21 / 2011$ \\
\hline 59 & $6 / 11 / 2010$ & $6 / 14 / 2010$ & $6 / 15 / 2010$ & $6 / 16 / 2010$ & 450 & 5 & $1 / 21 / 2011$ \\
\hline 60 & $6 / 10 / 2010$ & $6 / 14 / 2010$ & $6 / 15 / 2010$ & $6 / 16 / 2010$ & 450 & 6 & $1 / 21 / 2011$ \\
\hline 61 & $6 / 10 / 2010$ & $6 / 14 / 2010$ & $6 / 15 / 2010$ & $6 / 16 / 2010$ & 450 & 6 & $1 / 21 / 2011$ \\
\hline 85 & 6/9/2010 & $6 / 10 / 2010$ & $6 / 11 / 2010$ & $6 / 11 / 2010$ & 450 & 2 & $1 / 21 / 2011$ \\
\hline 163 & $6 / 11 / 2010$ & $6 / 14 / 2010$ & $6 / 15 / 2010$ & $6 / 16 / 2010$ & 450 & 5 & $1 / 21 / 2011$ \\
\hline 195 & $6 / 14 / 2010$ & $6 / 17 / 2010$ & $6 / 18 / 2010$ & $6 / 18 / 2010$ & 450 & 4 & $1 / 21 / 2011$ \\
\hline 213 & 6/8/2010 & $6 / 10 / 2010$ & $6 / 11 / 2010$ & $6 / 11 / 2010$ & 450 & 3 & $1 / 21 / 2011$ \\
\hline 227 & $6 / 8 / 2010$ & $6 / 10 / 2010$ & $6 / 11 / 2010$ & $6 / 11 / 2010$ & 450 & 3 & $1 / 21 / 2011$ \\
\hline 277 & $6 / 9 / 2010$ & $6 / 10 / 2010$ & $6 / 11 / 2010$ & $6 / 11 / 2010$ & 450 & 2 & $1 / 21 / 2011$ \\
\hline 299 & $6 / 8 / 2010$ & $6 / 10 / 2010$ & $6 / 11 / 2010$ & $6 / 11 / 2010$ & 450 & 3 & $1 / 21 / 2011$ \\
\hline 379 & $6 / 11 / 2010$ & $6 / 14 / 2010$ & $6 / 15 / 2010$ & $6 / 16 / 2010$ & 450 & 5 & $1 / 21 / 2011$ \\
\hline 507 & $6 / 15 / 2010$ & $6 / 17 / 2010$ & $6 / 18 / 2010$ & $6 / 18 / 2010$ & 450 & 3 & $1 / 21 / 2011$ \\
\hline 42133 & $6 / 15 / 2010$ & $6 / 17 / 2010$ & $6 / 18 / 2010$ & $6 / 18 / 2010$ & 450 & 3 & $1 / 21 / 2011$ \\
\hline
\end{tabular}

${ }^{1}$ Date sediment sample collected from the field.

${ }^{2}$ Date sediment sample was shipped to U.S. Geological Survey laboratory in Corpus Christi, Texas.

${ }^{3}$ Date sediment sample was received at the U.S. Geological Survey laboratory in Corpus Christi, Texas.

${ }^{4}$ Date pore water was extracted from the sediment and frozen.

${ }^{5}$ Volume of pore water extracted and frozen (milliliters).

${ }^{6}$ Number of days between sample collected in the field and porewater extraction and freezing.

${ }^{7}$ Date that salinity adjusted pore water was tested. 


\section{Appendix 3}

Date Prepared: May 5, 1990

Date Revised: July 18, 2007

\section{EXTRACTION AND STORAGE OF PORE-WATER SAMPLES}

\subsection{OBJECTIVE}

This protocol describes a procedure for extracting and storing pore-water samples from marine, estuarine, or freshwater sediments for use in toxicity testing. A pressurized extraction device is used to force the pore water from sediment samples. This procedure may be performed in the laboratory or it may be performed at or near the site of sample collection since the sampling apparatus is portable.

\subsection{PREPARATION}

\subsection{Description of the Pore-water Extraction System}

In earlier studies (Carr and others, 1989; Carr and Chapman, 1992) pore water was extracted from sediments using a device constructed of Teflon ${ }^{\circledR}$. Since then, the design has been improved (Carr and Chapman, 1994). The polyvinyl chloride (PVC) extractors in current use are less costly to construct and easier to operate. This device has been used in numerous sediment quality assessment surveys (Carr, and others, 1996a, 1996b, 1996c, 2000, 2001).

The extractor is constructed from a PVC compression coupling for 4" I.D. schedule 40 PVC pipe. These commercially-available couplings (Lascotite ${ }^{\circledR}$ ) consist of a cylinder (25 centimeter $(\mathrm{cm})$ height and 13 centimeter diameter) with threaded ends and threaded open compression nuts (fig. 1-1). The coupling is fitted with end plates cut from $7 / 16$ inch thick PVC sheeting that is held in place by the threaded end nuts. The gaskets provided with the coupling are discarded and silicon O-rings are used to seal the top and bottom connections. The top end plate is fitted with a quick-release fitting where the pressurized air is supplied, and a safety pressure relief valve. Like the original Teflon ${ }^{\circledR}$ extractor, the bottom end plate (fig. 1-1) has several interconnected concentric grooves to facilitate flow of the pore water to the central exit port. A 5-micrometer polyester filter is situated between the bottom end plate and the silicon O-ring. Before a sediment sample is loaded, the bottom end nut is tightened in place by using the stationary bottom wrench (fig. 1-1) and a standard strap wrench. 


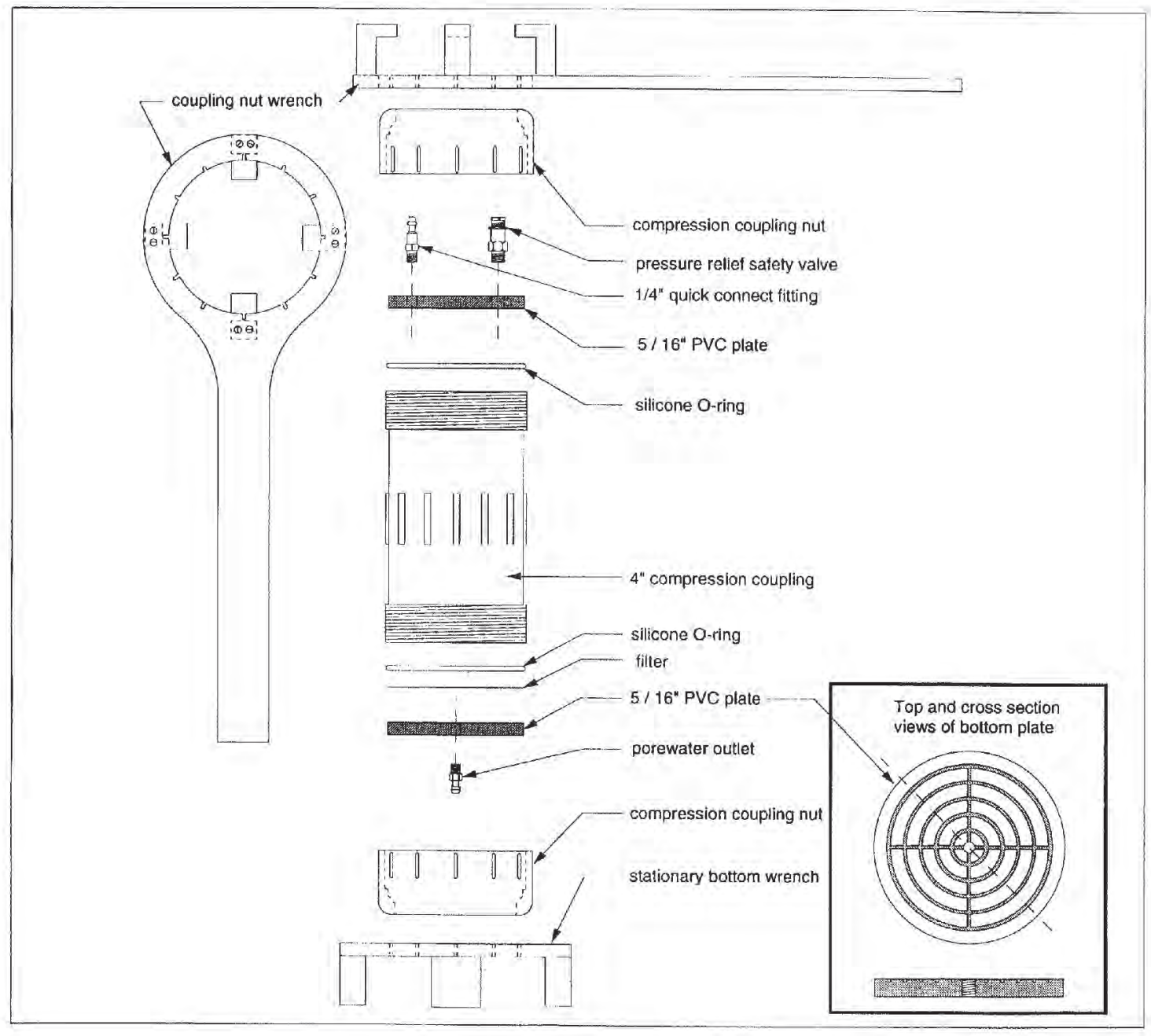

Figure 1-1. Sediment pore water squeeze extraction device. 
The extractors are pressurized with air supplied from a standard SCUBA cylinder via a SCUBA first stage regulator which delivers air to a manifold with a valving system (fig 1-2). With this system, multiple cylinders can be pressurized simultaneously, using the same SCUBA cylinder.

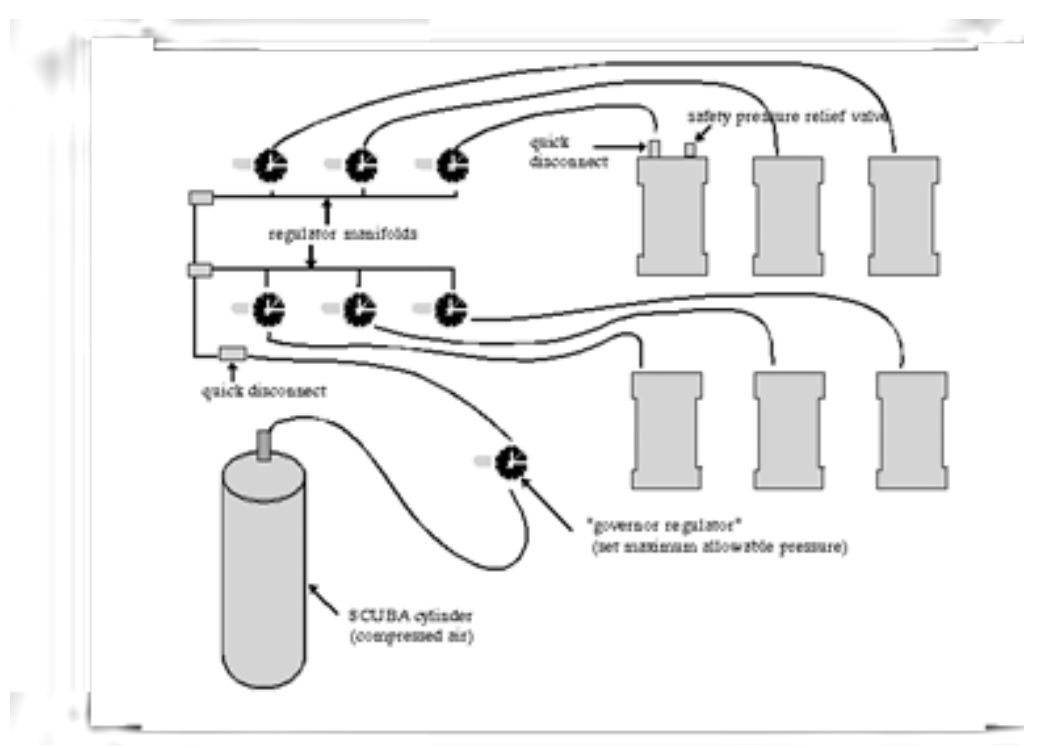

Figure 1-2. Schematic of sediment pore-water pressure extraction system.

\subsection{Equipment List}

Supplies and equipment needed are listed in Attachment A.

\subsection{PROCEDURE}

\subsection{Sediment Collection and Storage Considerations}

Generally, surficial sediment samples are collected for pore-water extraction. A homogenate of the upper $-2-10 \mathrm{~cm}$ sediment may be collected by multiple cores or grabs at a particular sampling station. (Further details of sediment sampling procedures are not within the scope of this SOP.) One liter of sediment will typically provide 100-200 milliliters (mL) pore water. However, a larger volume of coarse sand sediments may be required since they contain less water, and a larger volume of fine clay sediments may be required since they are difficult to extract. The sample composites are kept in suitable containers (e.g., clean high density polyethylene containers or Zip-Lock ${ }^{\circledR}$ bags), labeled, and stored on ice, in a cooler, or in a refrigerator until the samples are delivered and processed. Pore water should be extracted from the samples as soon as possible because the toxicity of sediments in storage may change over time. A sample tracking system should be maintained for each sediment sample collected and pore-water sample extracted. All manipulations made on samples are recorded on the Sample History Data Form (Attachment B). 


\subsection{Load Extraction Cylinder}

1. Assemble all parts of extraction cylinder except the top end compression coupling nut, top end plate and O-ring. Make sure filter is snugly in place beneath bottom O-ring (both overand under-tightening will result in an improper seal). Place the extractor cylinder on the stand and position an appropriately labeled pore-water sample container (usually an certified pre-cleaned amber $250 \mathrm{~mL}$ or $125 \mathrm{~mL}$ glass jar cleaned to U.S. Environmental Protection Agency standards, with Teflon ${ }^{\circledR}$ lid liner) underneath the outlet.

2. Ensure that the sediment sample is homogenized, by shaking, stirring with a clean Teflon ${ }^{\circledR}$ or plastic spatula or spoon, or by both.

3. Transfer sediment from the sample container/bag to the extractor by pouring and/or using a clean Teflon ${ }^{\circledR}$ or plastic spatula or spoon. If necessary, particularly when extracting pore water from sandy or shelly sediments, the spatula may be used to compress the sample in the cylinder to eliminate channelization. The amount of sediment to be transferred will depend on the texture of the sample. The cylinder may be filled nearly full with sandy sediment. However, when extracting pore water from clay sediment, a relatively impermeable layer of compressed clay will eventually form on the filter, so that extraction of a large volume of clay sediment at once would take an extremely long time. When extracting pore water from extremely fine grained sediments, the cylinder should be less than one-third filled. If additional pore water is needed, this process can be repeated by removing the sediment including removing or "peeling" the impermeable layer, and reintroducing more of the original sediment sample.

4. After sediment is loaded, the top end plate within the top compression coupling nut is installed. To tighten the top nut, the strap wrench and the coupling nut wrench (fig. 1-1) are used.

\subsection{Pore-water Extraction}

After the extractor is sealed, a high-pressure hose is attached to the quick disconnect fitting on the top end plate, and the extractor is pressurized with air from a SCUBA tank. Pressure is controlled with a first-stage regulator on the SCUBA tank, an intermediate "governor" regulator, and final second stage regulators attached to a manifold that services multiple extractors (fig. 1-2).

1. Turn the SCUBA valve counter clockwise, pressurizing the first stage regulator and the intermediate-pressure hose (approximately 150 pounds per square inch; psi). An additional "governor" pressure regulator between the SCUBA tanks and the final second stage regulators which control pressure to the individual extractors should be set at maximum extractor pressure (-40 psi).

2. Ensure that all final pressure regulators are set to zero. Attach the hose from one of the pressure regulators on the pressure regulator manifold to the air inlet, using the quick disconnect fitting. 
3. Slowly open the corresponding pressure regulator to a pressure of 5-10 psi. Check the first drops of pore water passing from the outlet for cloudiness. Occasionally, a small amount of sediment will pass through the pore-water outlet, presumably around the filter. If this happens, wait until the pore water clears, discard the initial pore water collected, and continue.

4. Check the cylinder for leaks and if necessary tighten clamping nuts slightly.

5. As the flow of pore water decreases, pressure may be increased gradually to a maximum of 35-40 psi. When flow is less than or slows to less than 1-3 drops per minute, increase the pressure in 5-10 psi increments to maintain the flow. Allow the extraction to continue until sufficient pore water has been collected.

6. Disassemble the extractor, discard sediment, and rinse and wash appropriately all parts contacting sediment before placing a different sediment sample into the extractor.

7. Repeat these procedures until all available extractors are in use or until all sediment samples have been processed.

\subsection{Centrifugation of Pore-water Samples}

Pore-water samples extracted at this field station are usually stored frozen until tested. Under most circumstances, the pore-water samples are centrifuged after they are collected and before they are frozen.

1. After collection, keep the pore-water samples refrigerated or chilled on ice until they are centrifuged.

2. Transfer the pore water from the glass sample jar to an appropriate centrifuge bottle (e.g., polycarbonate). Centrifuge at greater than or equal to 1,200 times gravity $(g)$ for 20 minutes. Return the centrifuged sample to a rinsed and labeled glass jar, taking care not to disturb any material that may have settled on the bottom/sides of the centrifuge bottle.

3. If multiple jars of pore water were collected from a single sediment sample, they should be composited after centrifugation and redistributed to the glass jars before testing or storage.

\subsection{Storage of Pore-water Samples}

If the pore-water samples are not to be used on the day of collection, they should be frozen for storage. Sufficient room for freeze expansion should be left in the jars (for example, $200 \mathrm{~mL}$ maximum sample in a $250 \mathrm{~mL}$ jar). If the volume needed for testing is known in advance, it is prudent to allocate only that specific volume plus a little excess $(-10 \mathrm{~mL})$ to each jar in order to conserve pore water (once thawed, the pore water cannot be refrozen and reused), and to simplify the volume measurements required for Water Quality Adjustment of Samples (CERC SOP P.651) performed the day prior to testing. Frozen pore-water samples may be shipped with dry ice. 


\subsection{QUALITY CONTROL}

A sample tracking system is maintained for each sediment sample collected and pore-water sample extracted. All actions taken with that respective sample are recorded on the Sample History Data Form (Attachment B). This information includes, but not exclusively, : a) the date of collection or receipt, b) the date of pore-water extraction, c) the volume or number of jars (certified pre-cleaned amber glass jars) of pore water collected, d) centrifugation information, if performed, e) date frozen and location (freezer number), and e) date and jar no. thawed and used in which test. The Sample History Forms are kept in a three-ring binder at the same location where the samples are stored.

\subsection{TRAINING}

Persons who will perform this procedure should first read this SOP and then operate under the supervision of an experienced individual for at least one series of extractions.

\subsection{SAFETY}

The sediment and pore-water samples handled may contain contaminants. Care should be taken to avoid contact with the samples. Protective gloves, glasses and clothing may be worn. Waste sediment should be properly disposed. SCUBA cylinders should be securely mounted before, during, and after use. The pressure limit (40 psi) of the extraction cylinders should not be exceeded. Before disconnecting any pressure hoses, ensure that the pressure has been released or that the controlling regulator has been closed.

\subsection{ATTACHMENTS}

Attachment A. Required Equipment and Materials

Attachment B. Sample History Form

\subsection{REFERENCES}

Carr, R.S., Chapman, D.C., Howard, C.L., and Biedenbach, J.M., 1996a, Sediment Quality Triad assessment survey in the Galveston Bay Texas system: Ecotoxicology, v. 5, p. 341-361.

Carr, R.S., Chapman, D.C., Presley, B.J., Biedenbach, J.M., Roberton, L., Booth, P., Kilada, R., Wade, T., and Montagna, P.,1996b, Sediment pore-water toxicity assessment studies in the vicinity of offshore oil and gas production platforms in the Gulf of Mexico: Canadian Journal Aquatic Fisheries Science, v. 53, p. 2,618-2,628.

Carr, R.S., Long, E.R., Chapman, D.C., Thursby, G., Biedenbach, J.M., Windom, H., Sloane, G., and Wolfe, D.A., 1996c, Toxicity assessment studies of contaminated sediments in Tampa Bay, Florida: Environmental Toxicology and Chemistry, v. 15, p. 1,218-1,231. 
Carr, R.S., Montagna, Biedenbach, J.M., Kalke, R., Kennicutt, M.C., Hooten, R., and Cripe, G., 2000, Impact of storm water outfalls on sediment quality in Corpus Christi Bay, Texas: Environmental Toxicology and Chemistry, v. 19, p. 561-574.

Carr, R.S., Biedenbach, J.M., and Hooten, R., 2001, Sediment pore-water toxicity test survey and phase I sediment toxicity identification evaluation studies in Lavaca Bay, Texas - an estuarine Superfund site: Environmental Toxicology, v. 16, p. 20-30. 
Prepared by:

James M. Biedenbach

Biologist

Approved by:

R. Scott Carr

Field Station Leader

Laverne Cleveland

Chief, Field Stations Research Branch

Paul Heine

Quality Assurance Officer 
$\underline{\text { Attachment A }}$

\section{REQUIRED EQUIPMENT AND MATERIALS}

To construct a sediment pore water extraction device:

1-PVC cylinder (center portion of 4 inch compression coupling)

2-PVC end nuts (ends of 4 inch compression fitting)

1-PVC top end plate (7/16 inch width)

1-PVC bottom end plate (7/16 inch width)

1-Quick disconnect brass air fitting

1-Pressure relief valve

1 -Teflon ${ }^{\circledR} 1 / 8$ inch national pipe thread (npt) male connector for exit port

To use a pore water extraction device:

1-Filter, polyester material, $5 \mu \mathrm{m}$ pore size

1 -Wooden stand ( 1 stand per 3 cylinders)

1-Custom wrench for 4 inch compression coupling end nuts

1-Custom wrench head attached to table

1-Plastic or Teflon ${ }^{\circledR}$ spatula or spoon

1-SCUBA cylinder

1-SCUBA regulator with high pressure gauge

1-SCUBA intermediate pressure hose (-10 ft length)

with governor pressure gauge set to $-40 \mathrm{psi}$

1-Air pressure control manifold that includes:

Final pressure regulator valves (several per manifold)

Pressure gauges ( 1 per valve)

Low pressure hose, 6 foot length (1 per manifold)

Other required supplies/equipment:

Sediment sample containers or bags

Pore water sample jars

Sample labels or labeling tape

Beakers

Deionized water (DI)

Wash bottles, $500 \mathrm{ml}$

Protective gloves, glasses, clothing

Pens, pencils, markers

Centrifuge and centrifugation materials

Refrigerator

Freezer 
$\underline{\text { Attachment B }}$

\section{SAMPLE HISTORY DATA FORM}

Sample Designation: Study Protocol: Initials:

Date of acquisition: Sample type:

How acquired (refer to sample site data sheet number, if appropriate):

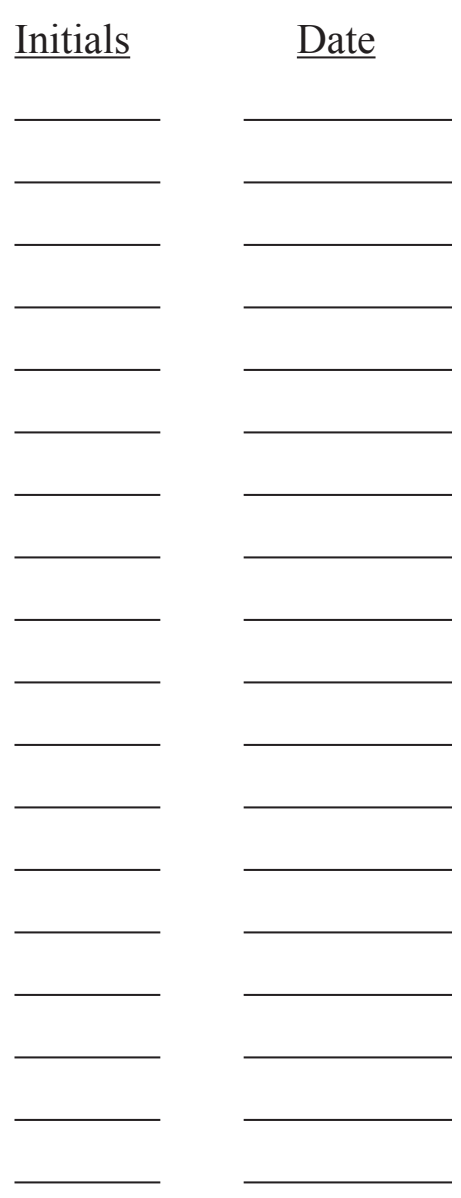

$\underline{\text { Action Taken }}$ 


\section{Appendix 4}

Date Prepared: March 14, 1991

Date Revised: July 17, 2007

\section{WATER QUALITY ADJUSTMENT OF SAMPLES}

\subsection{OBJECTIVE}

In order to perform toxicity tests with saline samples, all test and reference samples should be similar in salinity so that salinity is not a factor in survival of test organisms. Additionally, dissolved oxygen (DO) concentrations should be sufficiently high to ensure that low DO is not a source of stress to the test organisms. At the Corpus Christi field station, toxicity tests are performed using a variety of marine and estuarine organisms, including the sea urchin Arbacia punctulata, the polychaete Dinophilus gyrociliatus, the harpacticoid copepod Schizopera knabeni, and the red drum Sciaenops ocellatus. The aqueous samples tested may be pore water, different kinds of discharges and effluents, surface microlayer, or subsurface water samples that may range in salinity from 0-36 parts per thousand (\%o). Although salinities used in the different toxicity tests may vary, the individual toxicity tests performed on a particular day are run at one target salinity. Since initial salinities of the porewater or water samples to be tested commonly vary, they will require salinity adjustment to within $1 \%$ of the target salinity. Additionally, DO should normally be greater than or equal to 80 percent saturation in all samples tested.

\subsection{PREPARATION}

\subsection{Equipment and Labware}

The supplies and equipment needed are listed in Attachment 1.

\subsection{Source of Dilution Water}

For samples lower in salinity than target salinity, concentrated brine $(-100 \%)$ is added to increase salinity. Concentrated brine is prepared by heating (to $35-40{ }^{\circ} \mathrm{C}$ ) and gently aerating filtered natural seawater $(1$ micrometer $(\mu \mathrm{m}))$ to concentrate the salts by evaporation. For samples higher in salinity than target salinity, Millipore Milli- $\mathrm{Q}^{\circledR}$ ultrapure water is added to decrease salinity.

\subsection{P ROCEDURES}

The following describes the procedures required for the adjustment and determination of specific water quality parameters of a sample. 


\subsection{Preparation for Salinity Adjustment}

1. Although fresh samples are routinely tested at the Corpus Christi field station, most of the samples tested are stored frozen in certified pre-cleaned amber jars. If frozen, remove samples from freezer and allow them to thaw at room temperature or immerse them in a tepid water bath to thaw, ensuring that sample temperature does not exceed $25^{\circ} \mathrm{C}$. The samples may be thawed the day of water quality adjustment (WQA) or may be transferred from the freezer to a refrigerator $\left(4^{\circ} \mathrm{C}\right)$ the day before WQA and then completely thawed the following day. After thawing, allow the samples to come to room temperature. Generally, the samples should be maintained at the same temperature required for the toxicity test that will be conducted. The temperature requirement for most toxicity tests performed at this field station is $20 \pm 1{ }^{\circ} \mathrm{C}$, and room temperature should be maintained accordingly.

2. Turn bottled sample end over end a few times to mix thoroughly before measuring salinity. Using a refractometer, measure salinity and record on Water Quality Adjustment Data Form (Attachment 2).

3. In order to make calculations for the salinity adjustment, the volume of the sample must be known. When porewater or other water samples are collected and transferred to amber jars for storage, they are commonly measured to an approximate volume $(-110 \mathrm{~mL}$, for example) prior to freezing. On the day of WQA, this volume should be recorded on the WQA data form for the respective samples. If the volume is unknown at this point, it should be measured using a graduated cylinder of appropriate size, and recorded on the data sheet.

\subsection{Salinity Adjustment}

\subsubsection{Reducing the salinity of aqueous samples}

Refer to the formulas below to calculate the volume of Milli- ${ }^{\circledR}$ water needed to reduce the initial sample salinity to the target salinity. Add the volume calculated, mix the bottle thoroughly, check the salinity with a refractometer, and record the volume of Milli-Q ${ }^{\circledR}$ water added as well as the final salinity.

(i) (Target $\% \circ \div$ Sample \%o) x Sample volume in $m L=A$

(ii) Sample volume - $A=B$

(iii) Sample volume $\div A=C$

(iv) $\quad B \times C$-Volume of Milli- $Q^{\circledR}$ water to add 


\subsection{Increasing the salinity of aqueous samples}

Refer to the formula below to calculate the volume of concentrated brine needed to increase the initial sample salinity to the target salinity. Add the volume calculated, mix the bottle thoroughly, check the salinity with a refractometer, and record the volume of brine added as well as the final salinity.

$$
\text { (v.) } \frac{[(\text { Target } \% \text { - Sample } \%) \times \text { Sample volume in } m L]}{(\text { Brine } \% \text { - Target } \%)}=\text { Volume of brine to add }
$$

\subsection{Dissolved Oxygen Adjustment}

Measure and record DO and percent DO saturation of sample (SOP P.652). Occasionally, a sample will have DO of less than 80 percent saturation. Any such samples should be gently stirred on a magnetic stirrer to increase the DO level above 80 percent. Record initial DO, the elapsed mixing time, and final DO in the comments section of the Water Quality Adjustment Data Form. (On the following day, DO should be rechecked and brought to greater than 80 percent saturation by stirring again if necessary before the toxicity test is performed.)

\subsection{Other Water Quality Determinations}

1. Measure $\mathrm{pH}$ (SOP P.658) and record on the Water Quality Adjustment Data Form (Attachment 2).

2. Measure and record ammonia concentration (SOP P.646).

3. Measure and record sulfide concentration if required (SOP P.657).

\subsection{DATA COLLECTION}

All raw data are entered on one standardized form, the Water Quality Adjustment Data Form (see Attachment 2) at the time the determinations or adjustments are made.

\subsection{QUALITY CONTROL}

A data form (Attachment 2) will be used to document all sample handling procedures for each sample. The person(s) recording data on the sheet will initial each sheet. Original data forms after completion will be stored in a three-ring file in the possession of the field station leader. Copies will be kept in the lab. 


\subsection{TRAINING}

Personnel who will perform this task should first read this protocol and then operate under supervision during the preparation of at least two samples.

\subsection{SAFETY}

The $\mathrm{NaOH}$ solution used in the ammonia determination procedure is a highly caustic liquid. Care should be taken to avoid its contact with skin or clothing. Should such contact occur, quickly flush affected with water. Multiple sinks are present in each of the labs, and an eye flushing station and emergency shower is present near the entrance door of each lab. The samples handled may be pore water, effluent, discharges, or other water samples that may contain contaminants. Care should be taken to avoid contact with the samples by donning the appropriate safety clothing and gloves.

\subsection{ATTACHMENTS}

Attachment 1. Equipment List for Water Quality Adjustment

Attachment 2. Water Quality Adjustment Data Form 
32 Sediment Toxicity Test Results for the Urban Waters Study 2010, Bellingham Bay, Washington

Prepared by:

James M. Biedenbach

Biologist

Approved by:

R. Scott Carr

Field Station Leader

Laverne Cleveland

Chief, Field Stations

Research Branch

Paul Heine

Quality Assurance Officer 


\section{$\underline{\text { Attachment } 1}$}

\section{EQUIPMENT LIST FOR WATER QUALITY ADJUSTMENT}

Graduated cylinders

Pipetters

Latex gloves

Magnetic stirrer and stir bars

$10 \mathrm{M} \mathrm{NaOH}$

Concentrated brine (See section 2.2 for preparation)

Milli-Q ${ }^{\circledR}$ ultrapure water

Salinity refractometer

Dissolved oxygen meter

$\mathrm{pH}$ electrode, buffer solutions, and meter

Ammonia electrode, standard solutions, and meter

Sulfide electrode, standard solutions, and meter

Data sheets

Hand calculator 


\section{Attachment 2}

\section{WATER QUALITY ADJUSTMENT DATA FORM}

STUDY PROTOCOL

SAMPLE DESIGNATION
INITIALS

DATE

A. Salinity Adjustment:

Initial volume $(\mathrm{mL})$

Initial salinity (\%o)

Vol. Milli-Q ${ }^{\circledR}$ water added (mL)

Vol. _ \%o brine added $(\mathrm{mL})$

Percent of original sample

(initial volume/final volume $\times 100$ )

B. Character of Sample (after salinity adjustment):

Volume $(\mathrm{mL})$

Salinity (\%)

$\mathrm{pH}$

Dissolved oxygen $(\mathrm{mg} / \mathrm{L})$

DO saturation (percent)

Total ammonia $(\mathrm{mg} / \mathrm{L})$

Sulfide $(\mathrm{mg} / \mathrm{L})$ 


\section{Appendix 5}

Date Prepared: July 21, 1989

Date Revised: July 24, 2007

\section{MEASURING DISSOLVED AMMONIA WITH THE THERMO ORION ${ }^{\circledR}$ MODEL 95-12 AMMONIA PROBE}

\subsection{APPLICATION}

The ammonia electrode is used to measure the concentration of ammonia in aqueous media. The detectable concentration range is from 0.01 to 14,000 milligrams per liter ( $\mathrm{mg} / \mathrm{L}$ ) ammonia nitrogen, although in our laboratory concentrations measured usually fall within the range of 0.1 to $100 \mathrm{mg} / \mathrm{L}$.

\subsection{OPERATION}

\subsection{Required Equipment and Solutions}

1. Thermo Orion ${ }^{\circledR}$ model $95-12$ ammonia probe

2. Thermo Orion ${ }^{\circledR}$ model 290A pH/ISE meter

3. Magnetic stirrer

4. Bonded membrane caps (Thermo Orion ${ }^{\circledR}$ \#951205) or loose membranes (Thermo Orion $\left.^{\circledR} \# 951204\right)$

5. Electrode internal filling solution (Thermo Orion ${ }^{\circledR} \# 951202$ )

6. Standardizing Solution, $\mathrm{NH}_{4} \mathrm{Cl}, 1000 \mathrm{mg} / \mathrm{L}$ as nitrogen (Thermo Orion ${ }^{\circledR}$ \#951007) or prepare as described below.

7. 10 Molar (M) NaOH

\subsection{Electrode Preparation and Assembly}

\subsubsection{Electrode Setup}

When the electrode is first received or after it has been stored dry, soak the inner body in internal filling solution for at least two hours before assembling the electrode. For best results, soak the inner body overnight. Then, follow these steps:

1. Unscrew top cap and remove glass electrode inner body, and drain old filling solution. Set cap with inner body aside carefully.

2. Remove bottom cap from electrode outer body. If using bonded membrane caps, screw cap in end of electrode until finger-tight, and proceed to step 5. If using loose membranes, proceed with instructions in step 3 . 
3. Using tweezers, carefully grasp a white membrane from between paper separators. Hold membrane at the edge with the tweezer. Holding electrode outer body in free hand, loosely stretch the membrane across opening in bottom of probe, holding the ends against the threads with your thumb and forefinger. Avoid excessive handling of the membrane since this may affect its hydrophobic properties and reduce its life.

4. Replace cap onto probe and screw until finger-tight. The membrane should be smooth with no wrinkles.

5. Add $2.5 \mathrm{ml}$ internal filling solution into the electrode outer body. If measuring low ammonia concentrations (eg. $<0.06 \mathrm{mg} / \mathrm{L}$ ammonia nitrogen), the filling solution can be diluted by $1 / 10$ to increase response time.

6. Replace inner body into outer body and screw on upper cap.

7. Shake fully assembled electrode as if it were a clinical thermometer to remove bubbles.

8. Record date of membrane and internal filling solution change in instrument record/equipment log.

9. Soak the assembled probe in $10 \mathrm{mg} / \mathrm{L} \mathrm{NH}_{4} \mathrm{Cl}$ standard for at least 2 hours before making measurements.

\subsubsection{Checking Electrode Operation}

Obtaining the slope value (the change in $\mathrm{mV}$ observed with every tenfold change in concentration) provides the best means for checking electrode operation. If using the model 290A meter and problems have not been encountered, proceed to step 6 .

1. Place $100 \mathrm{~mL}$ deionized (DI) water into a $150 \mathrm{~mL}$ beaker. Add $1 \mathrm{~mL} 10 \mathrm{M} \mathrm{NaOH}$ and stir thoroughly. (The $\mathrm{NaOH}$ addition raises the $\mathrm{pH}$ above $\mathrm{pH} 11$ where all of the ammonia present is in the unionized form.) Set the function switch of the meter to the $\mathrm{mV}$ mode.

2. Rinse electrode with DI water and place in the solution prepared in step 1.

3. Pipet $1 \mathrm{~mL}$ of the $1,000 \mathrm{mg} / \mathrm{L}$ ammonium chloride $\left(\mathrm{NH}_{4} \mathrm{Cl}\right)$ standard into the beaker and stir thoroughly. Record the electrode potential in millivolts $(\mathrm{mV})$.

4. Pipet $10 \mathrm{~mL}$ of the same $1,000 \mathrm{mg} / \mathrm{L} \mathrm{NH}_{4} \mathrm{Cl}$ standard into the same beaker and stir thoroughly. Again, record the electrode potential.

5. The difference between the first and second potential reading is the slope of the electrode. The electrode slope should fall within the range of negative 54 to negative $60 \mathrm{mV}$ when the temperature of the solution is $20-25^{\circ} \mathrm{C}$. Note the electrode slope in the instrument records/equipment log. If the slope is not within this range, check 
meter operation, ensure that the electrode is properly prepared and assembled, or refer to section 4.3 Troubleshooting.

6. Alternatively, the slope can be viewed after performing the normal calibration procedures undertaken before making measurements (Section 2.4 Perform Calibration Using Standard Solutions). Using the Model 290A meter (Orion $\left.{ }^{\circledR}\right)$, the slope value is flashed on the LCD display after the "measure" button on the keypad is first depressed. Also, the slope can be reviewed at any time, following calibration, in the "setup" menu under category $2-1$.

\subsection{Preparation of Standards}

1. A $1,000 \mathrm{mg} / \mathrm{L}$ ammonia as nitrogen stock solution can be purchased (Thermo Orion ${ }^{\circledR}$ \#951007) or prepared. Prepare by adding 3.82 g reagent-grade $\mathrm{NH}_{4} \mathrm{Cl}$ to $500 \mathrm{~mL}$ DI water in a $1,000 \mathrm{~mL}$ volumetric flask, stir to dissolve and dilute to volume with DI water. Make or purchase again before shelf life expires (-6 months).

2. Prepare $100,10,1.0$, and $0.1 \mathrm{mg} / \mathrm{L}$ standard solutions by serial dilution from the $1,000 \mathrm{mg} / \mathrm{L}$ stock solution using 0.45 micrometer $(\mu \mathrm{m})$ filtered seawater $(\mathrm{SW})$. These can be made in scintillation vials:

$$
\begin{aligned}
& 1 \mathrm{~mL} 1,000 \mathrm{mg} / \mathrm{L} \text { standard }+9 \mathrm{~mL} \mathrm{SW}=100 \mathrm{mg} / \mathrm{L} \\
& 1 \mathrm{~mL} 100 \mathrm{mg} / \mathrm{L} \text { standard }+9 \mathrm{~mL} \mathrm{SW}=10 \mathrm{mg} / \mathrm{L} \\
& 1 \mathrm{~mL} 10 \mathrm{mg} / \mathrm{L} \text { standard }+9 \mathrm{~mL} \mathrm{SW}=1 \mathrm{mg} / \mathrm{L} \\
& 1 \mathrm{~mL} 1 \mathrm{mg} / \mathrm{L} \text { standard }+9 \mathrm{~mL} \mathrm{SW}=0.1 \mathrm{mg} / \mathrm{L}
\end{aligned}
$$

\subsection{Perform Calibration Using Standard Solutions}

1. Ensure that the mode is set to "concentration". For detailed instruction on how to operate the model 290A meter, see the Thermo Orion ${ }^{\circledR}$ Model 290A pH/ISE/Temperature/mV Meter instruction manual.

2. Run calibration using three standard solutions $(0.1,1.0$ and $10 \mathrm{mg} / \mathrm{L})$ beginning with the lowest concentration. Add 2 drops $10 \mathrm{M} \mathrm{NaOH}$ to $10 \mathrm{~mL}$ of each respective standard solution just prior to measuring. Ensure that each sample is fully stirred during measurement.

3. Check the slope (see item 6 in section 2.2.2 Checking Electrode Operation) and record in the comments section of the Water Quality Adjustment Data Form (Attachment 1) and the equipment log.

4. If concentrations $<0.1 \mathrm{mg} / \mathrm{L}$ are to be measured, another series of standard solutions for a lower range of values should be prepared, and another calibration should be performed.

5. Reproducibility of measurements, according to the instruction manual should be plus or minus \pm 2 percent with calibration performed every hour. Alternatively, the calibration can be verified every 1-2 hours by measuring a standard solution or internal reference solution. If the value is similar to that expected (within \pm 2 percent), recalibration is not necessary at 
that time. When verification and recalibration are undertaken, the data should be noted on the Water Quality Adjustment Data Form (Appendix 1).

\subsection{Measurements}

Following calibration, measure the concentration of at least one standard solution for verification. If the value is not within 2 percent, repeat the calibration procedures. Additionally, an internal reference sample (standard $\mathrm{NH}_{4} \mathrm{Cl}$ solutions previously prepared and frozen) may be measured as further verification. Once verification is complete, measure the various sample concentrations and record the data on the Water Quality Adjustment Data Form for each sample. As done with the standard solutions, 2 drops of $10 \mathrm{M} \mathrm{NaOH}$ should be added to $10 \mathrm{~mL}$ of each test sample just prior to measurement or 3 drops if the sample volume is $15 \mathrm{~mL}$.

\subsection{Measuring Hints}

1. The electrode should be rinsed with DI water between measurements.

2. The electrode should be checked for bubbles on the membrane. If present, they can be removed by shaking the electrode.

3. All samples should be magnetically stirred during measurement. Magnetic stirrers may generate some heat, and a layer of rigid foam can be placed below the sample to help limit any change in temperature.

4. It is critical that the temperature be consistent for all samples and standards. This ion selective electrode is not temperature compensated. A $1{ }^{\circ} \mathrm{C}$ difference will introduce a 2 percent measurement error. Water quality measurements should generally be made at the same temperature that the test will be carried out. At the U.S. Geological Survey laboratory in Corpus Christi, Texas, most testing is done at $20^{\circ} \mathrm{C}$. Consequently, room temperature while performing water quality measurements and the various toxicity tests should be maintained at $20^{\circ} \mathrm{C}$.

5. If electrode response is slow, the membrane may contain a surface layer of contaminants. To restore performance in this case, soak the electrode in DI water for -5 minutes and then soak in a standard solution for -1 hour before use or replace the membrane and soak in a $10 \mathrm{mg} / \mathrm{L}$ $\mathrm{NH}_{4} \mathrm{Cl}$ standard for -2 hours. For large numbers of samples, more then one probe may be assembled and utilized to reduce or eliminate the waiting time for probe equilibration.

6. If samples can not be measured on the same day they can be acidified with $1 \mathrm{M} \mathrm{HCl}$ to a $\mathrm{pH}$ of less then 2 and held refrigerated until the following day. 


\subsection{MAINTENANCE}

\subsection{Electrode Storage}

Between measurements, do not allow the electrode tip to dry. For overnight or week-long storage, the electrode tip should be immersed in a $1,000 \mathrm{mg} / \mathrm{L} \mathrm{NaOH}$-free ammonia standard. For storage over one week or if the electrode is stored indefinitely, disassemble completely and rinse the inner body, outer body, and bottom cap with DI water. Allow the parts to dry, and then reassemble the electrode without internal filling solution or a membrane.

\subsection{Membrane Life}

According to the instruction manual, membranes will last from 1 week to several months depending on usage. At the USGS laboratory, Corpus Christi, TX, membranes may last less than one day when measuring samples with contaminants or high ammonia levels. Membrane failure is characterized by a shift in electrode potential, drift, and poor response. Membrane failure may be apparent on visual inspection as dark spots or discoloration of the membrane or carbonate deposits on the membrane and end cap. Follow the procedures given in section 2.2.1 Electrode Setup to replace the membrane and add internal filling solution.

\subsection{Troubleshooting}

Generally, if the meter is operating properly, and if the electrode has been prepared and assembled properly and has passed the electrode checkout procedures, the electrode slope should fall within the range of negative 54 to negative $60 \mathrm{mV}$ when the temperature of the solution is $20-25^{\circ} \mathrm{C}$. When the temperature of the solution is $20^{\circ} \mathrm{C}$, the electrode slope should be approximately negative $58.2 \mathrm{mV}$. For problems encountered that this SOP does not address, refer to the Model 290A meter instruction manual (Thermo Orion ${ }^{\circledR}$, Inc. 1991) and the Model 95-12 ammonia electrode instruction manual (Thermo Orion ${ }^{\circledR}$, Inc. 2002).

\subsection{TRAINING}

Personnel who will perform this task should first read this protocol and then operate under supervision until proper technique and accuracy of measurements is ensured.

\subsection{SAFETY}

$10 \mathrm{M} \mathrm{NaOH}$ is a highly caustic liquid. Care should be taken to avoid its contact with skin or clothing. Should such contact occur, quickly flush affected area with water. Sinks are present along 2 walls of the wet lab, and an eye flushing station and shower is present near the entrance door of all labs. The samples handled may be pore water, effluent, discharges, or other water samples that may contain contaminants. Care should be taken to avoid contact with the samples by donning gloves, lab coats and other necessary safety equipment. 


\subsection{ATTACHMENTS}

Attachment 1. Water Quality Adjustment Data Form

\subsection{REFERENCES}

Thermo Orion ${ }^{\circledR}$ Inc., 1991, Portable pH/ISE Meters Instruction Manual (Models 230A, 250A, and 290A): Beverly, MA., Thermo Electron Corporation, 60 p.

Thermo Orion ${ }^{\circledR}$ Inc., 2002, Model 95-12 Ammonia Electrode Instruction Manual: Beverly, MA., Thermo Electron Corporation, $42 \mathrm{p}$. 
Revised by:

James M. Biedenbach

Biologist

Approved by:

R. Scott Carr

Field Station Leader

Laverne Cleveland

Chief, Field Stations Research Branch

Paul Heine

Quality Assurance Officer 


\section{$\underline{\text { Attachment } 1}$}

\section{WATER QUALITY ADJUSTMENT DATA FORM}

STUDY PROTOCOL

SAMPLE DESIGNATION
INITIALS

DATE

A. Salinity Adjustment:

Initial volume $(\mathrm{mL})$

Initial salinity (\%o)

Vol. Milli-Q ${ }^{\circledR}$ water added (mL)

Vol. __ \%o brine added (mL)

Percent of original sample

(initial volume/final volume $\times 100$ )

B. Character of Sample (after salinity adjustment):

Volume (mL)

Salinity (\%o)

$\mathrm{pH}$

Dissolved oxygen $(\mathrm{mg} / \mathrm{L})$

DO saturation (percent)

Total ammonia (mg/L)

Sulfide (mg/L) 


\section{Appendix 6}

Date Prepared: October 13, 1993

Date Revised: July 26, 2007

\section{MEASURING SULFIDE WITH THE THERMO ORION ${ }^{\circledR}$ MODEL 9616 SUREFLOW COMBINATION SILVER/SULFIDE PROBE}

\subsection{APPLICATION}

The silver/sulfide probe is used to measure the concentration of silver or sulfide ion in solution by measuring an electrode potential which develops across the sensing element. This potential, which depends on the level of free silver or sulfide ion in solution, is measured against a constant reference potential with a digital $\mathrm{pH} /$ millivolt $(\mathrm{mV})$ or specific ion meter.

\subsection{OPERATION}

\subsection{Required Equipment and Solutions}

1. Thermo Orion ${ }^{\circledR}$ model 9616 Sure-flow Combination silver/sulfide electrode

2. Thermo Orion ${ }^{\circledR}$ "A" Optimum Results ${ }^{\mathrm{TM}}$ Reference Electrode Filling Solution (\# 900061)

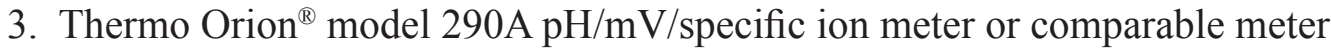

4. Sodium Sulfide stock solution (see 2.1.1)

5. Weekly Sulfide standard (see 2.1.2)

6. Standard sulfide solutions: $100,10,1,0.1, .08, .06, .05, .04, .03, .02, .0150, .0125, .01$, .009 milligrams per liter $(\mathrm{mg} / \mathrm{L})($ see 2.1 .3$)$

7. Magnetic stir bars and stirrer

8. Thermo Orion ${ }^{\circledR}$ Sulfide Antioxidant Buffer (SAOB II ${ }^{\circledR}$ ) solution (see 2.1.4 below)

9. De-aerated de-ionized (DI) water and de-aerated filtered seawater

\subsubsection{Sodium sulfide stock solution}

Prepare a stock solution of saturated sodium sulfide by dissolving approximately 100 g reagent grade $\mathrm{Na}_{2} \mathrm{~S}=9 \mathrm{H}_{2} \mathrm{O}$ in 100 milliliters $(\mathrm{mL})$ de-ionized, de-aerated water. Deionized water (DI) may be de-aerated by passing through a .45 micrometer $(\mu \mathrm{m})$ filter under vacuum suction. Shake the solution and let stand capped overnight in a fume hood.

\subsubsection{Weekly sufide standard}

Prepare a weekly sulfide standard by pipetting $1 \mathrm{~mL}$ of the stock solution into a $100 \mathrm{~mL}$ volumetric flask. Add $50 \mathrm{~mL}$ of SAOB $\mathrm{II}^{\circledR}$ solution and dilute to volume with de-ionized, de-aerated water. 
Determine the exact concentration, by titrating $10 \mathrm{~mL}$ of the standard with 0.1 molar (M) Lead Perchlorate, using the electrode pair as the end point indicator. Additions of Lead Perchlorate are best accomplished using a 1 cubic centimeter (CC) syringe with a fine needle. The end point is the point at which the $\mathrm{mV}$ reading drops rapidly with the addition of one more drop of Lead Perchlorate. In other words, if the $\mathrm{mV}$ readings were being graphed versus volume of Lead Perchlorate added, then the electrode pair would be the point on the graph with the greatest absolute slope.

Calculate the concentration $(C)$ of the weekly standard using the equation:

(i.) $\quad C=3206(V t \div V s)$ where:

$C=$ concentration as $\mathrm{mg} / \mathrm{L}$ sulfide

$V t=$ volume of Lead Perchlorate at end point $(\mathrm{mL})$

$V S=$ volume of standard titrated $(10 \mathrm{~mL})$

\subsubsection{Standard sulfide solutions}

Prepare other standards daily by dilution of the weekly standard with equal volumes of $0.45 \mu \mathrm{m}$ filtered, de-aerated seawater (FDS) and SAOB II. For example, to do a tenfold dilution, pipet $1 \mathrm{~mL}$ of the standard into a small beaker and add $4.5 \mathrm{~mL}$ of SAOB $\mathrm{II}^{\circledR}$ and $4.5 \mathrm{~mL}$ of FDS. It is recommended that the first dilution from the weekly stock solution be $100 \mathrm{mg} / \mathrm{L}$ followed by serial dilutions to achieve concentrations to the $0.1 \mathrm{mg} / \mathrm{L}$ concentration and then calculate the lower dilutions until the desired working range (ie. .009 to $10 \mathrm{mg} / \mathrm{L}$ ) is achieved. Remember: all dilutions must be made with both SAOB II ${ }^{\mathbb{B}}$ and FDS.

\subsubsection{SAOB II ${ }^{\circledR}$}

SAOB II ${ }^{\circledR}$ solution is commercially available from Thermo Orion ${ }^{\circledR}$ (\# 941609) or can be prepared by dissolving 40 grams (g) of Sodium Hydroxide and $29.2 \mathrm{~g}$ of Disodium Ethylenediamine Tetraacetate (EDTA) in de-aerated de-ionized water in a $500 \mathrm{~mL}$ volumetric flask. Prior to use this solution is "activated" by the addition of $18 \mathrm{~g}$ of Ascorbic acid. The activated solution will last up to one month if stored in a dark bottle.

\subsection{Instrument Setup}

1. Remove the rubber cap covering the electrode tip.

2. Fill the electrode with "A" Optimum Results ${ }^{\mathrm{TM}}$ reference electrode filling solution.

3. Connect the electrodes to the meter soak the probe in reference electrode filling solution for 10 minutes before calibrating the probe

\subsection{Calibration}

Measure $5 \mathrm{~mL}$ of the most dilute standard $(0.009 \mathrm{mg} / \mathrm{L})$ into a small beaker. Add $5 \mathrm{mLs}$ of SAOB II ${ }^{\circledR}$ solution and a stir bar and place on the automatic stirrer. Rinse electrodes, blot dry, and place in beaker. Wait for a stable millivolt reading and plot the reading (linear axis) 
against concentration (log axis) on standard 4-cycle semi-log paper. Repeat this process with the progressively higher concentration standards. Calculate the slope of the linear portion of the graph. The linear portion of the graft is that portion above $0.5 \mathrm{mg} / \mathrm{L}$. The slope of the curve should be between negative 25 and negative $30 \mathrm{mv} /$ decade. If the slope is out of range, perform the electrode slope check described in the probe instruction manual and/or consult the troubleshooting section of the manual.

\subsection{Sample Measurement}

Measure $5 \mathrm{~mL}$ of sample into a small beaker. Add $5 \mathrm{~mL}$ of SAOB II ${ }^{\circledR}$ solution and a stir bar and place on the automatic stirrer. Place probes in the solution and press the measure button on the meter. When the reading stabilizes, record the reading and read concentration from the calibration curve. Remove the probes, rinse with DI and blot. Continue with any additional samples.

Recheck the accuracy of the measurements by measuring the concentration of a known standard periodically. If the reading deviates more than 5 percent from the known value, recalibrate the probe.

\subsection{Sample Requirements and Measuring Hints}

1. Samples and standards should be at the same temperature. Temperature must be less than $100{ }^{\circ} \mathrm{C}$.

2. Sulfide samples must be buffered at $\mathrm{pH}$ greater than 12 with $\mathrm{SAOB}^{\mathrm{B}}{ }^{\circledR}$ so that $\mathrm{HS}^{-}$and $\mathrm{H}_{2} \mathrm{~S}$ are converted to $\mathrm{S}^{=}$.

3. Always use fresh standards for calibration mixed with de-aerated water.

4. Rinse electrodes with DI water between measurements and blot electrode dry.

5. For high ionic strength samples, (e.g., seawater) prepare standards with composition similar to that of sample (de-aerated seawater of similar salinity).

\subsection{MAINTENANCE}

1. If electrode response is slow, the sensing element may contain a surface layer of contaminants. Restore performance by polishing with polishing strips (included with the probe) and deionized water. Cut off 1-inch of the strip and polish the sensing element with a circular motion for 30 seconds. Rinse and soak in standard solution for 5 minutes before use.

2. The silver/sulfide probe should always be stored dry. The rubber cap should be replaced over the element during periods of long storage to protect it. 
3. The reference electrode may be stored dry or in internal reference filling solution for short periods of time (less than a week). For longer storage times, the solutions in the probes should be emptied and the chambers rinsed with DI to prevent crystallization.

\subsection{TRAINING}

Personnel who will perform this task should first read this Standard Operating Procedure (SOP) and operate the probe the first time under supervision.

\subsection{SAFETY}

SAOB II ${ }^{\circledR}$ is a caustic solution. Lead Perchlorate and Sodium Sulfide are irritants and toxic. Care should be taken and proper safety gear worn to avoid chemical contact with skin or clothing, or prolonged breathing of vapors. Should contact occur, flush the affected area with water and seek medical attention if warranted. An eye flushing station is present in the corner of the lab nearest the door, should it be needed.

\subsection{REFERENCES}

Thermo Orion ${ }^{\circledR}$ Inc., 1991, Portable pH/ISE Meters Instruction Manual (Models 230A, 250A, and 290A): Beverly, MA., Thermo Electron Corporation, 60 p.

Thermo Orion ${ }^{\circledR}$ Inc., 2001, Model 94-16 Silver/Sulfide Electrodes Instruction Manual: Beverly, MA., Thermo Electron Corporation, $67 \mathrm{p}$. 
Revised by:

James M. Biedenbach

Biologist

Approved by:

R. Scott Carr

Field Station Leader

Laverne Cleveland

Chief, Field Stations Research Branch

Paul Heine

Quality Assurance Officer 


\section{Appendix 7}

Date Prepared : September 19, 2006

Date Revised: $\quad$ May 10, 2007

\section{SEA URCHIN FERTILIZATION TOXICITY TEST WITH STRONGYLOCENTROTUS PURPURATUS}

\subsection{OBJECTIVE}

The purpose of the fertilization toxicity test with the sea urchin, Strongylocentrotus purpuratus, is to determine if seawater, pore water, sea surface microlayer, or other sample reduces fertilization of exposed gametes relative to that of gametes exposed to a control sample. The test may also be used to determine the concentration of a test substance which reduces fertilization. Test results are reported as treatment (or concentration) which produces significantly reduced fertilization or as concentration of test substance which effectively reduces fertilization by 50 percent $\left(\mathrm{EC}_{50}\right)$.

\subsection{TEST PREPARATION}

\subsection{Test Animals}

Gametes from the sea urchin, S. purpuratus are used in the sea urchin fertilization toxicity test. Animals can be collected in the field or obtained from a commercial supplier. S. purpuratus is a species of sea urchin found along the entire west coast of the United States and are characterized by a dark purple test with short light and dark purple spines. This species also has numerous tube feet between the rows of spines on both the dorsal and ventral surfaces of the test and often is found with algae, rocks and debris being held to the body with the tube feet. Due to the sensitivity of this species to the $\mathrm{pH}$ of Gulf Coast waters it is recommended to receive urchins for use in testing on the day of or the day before the actual test is to take place. If urchins are to be held overnight, they should be acclimated slowly to the temperature controlled aquarium water. Temperature of the cultures will prolong the useful life of the urchins and should be maintained at $12 \pm 1{ }^{\circ} \mathrm{C}$ when gametes are not required. Photoperiod is maintained at 16 hours of light per day. Water quality parameters should be monitored weekly and salinity maintained at $30 \pm 3$ parts per thousand (\%o). Males and females should be kept in separate tanks if the sex can be determined before use in testing.

\subsection{Dilution Water}

Milli- $Q^{\circledR}$ grade purified water or concentrated seawater brine is used to adjust samples to $30 \%$ as described in Water Quality Adjustment of Samples (CERC SOP P.651). Concentrated seawater brine (90-110\%) is made in large batches by heating seawater to $40{ }^{\circ} \mathrm{C}$ or less in large tanks with aeration for 3-4 weeks. Brine quality will remain constant over long periods with refrigeration. On the day of the test, $\mathrm{pH}$, ammonia, sulfide and dissolved oxygen are also measured. Salinity adjustment and water quality data are recorded on prepared data forms. 
Filtered $(0.45$ micrometer $(\mu \mathrm{m}))$ seawater adjusted to $30 \%$ is used to wash eggs and is also used for sperm and egg dilutions. The acronym MFS (for Millipore ${ }^{\circledR}$ filtered seawater) is used for this filtered and salinity adjusted seawater. Whenever possible, temperatures of wash, dilution and test waters should be maintained at test temperatures.

\subsection{Test System: Equipment}

When testing samples for potential toxicity, five replicates per treatment are recommended. One replicate is a 5 milliliters $(\mathrm{mL})$ volume of sample in a disposable glass scintillation vial. When conducting a dilution series test, fifty percent serial dilutions may be made in the test vials, using MFS as the diluent. For larger tests, (greater than 20 samples) it may be desirable to include control samples including dilutions at the beginning and at the end of the test to account for any variability due to decreased sperm viability during the pipetting process and provide for more statistical robustness for comparisons with a large number of samples.

\subsubsection{Equipment}

A list of equipment necessary for conducting this test is given in Attachment A (Equipment List for Fertilization Toxicity Test).

\subsubsection{Solutions}

$\underline{0.5 \text { molar }(\mathrm{M}) \text { Potassium Chloride }(\mathrm{KCl}) \text { solution: }}$

1. $3.73 \mathrm{~g} \mathrm{KCl}$ crystals

2. $100 \mathrm{~mL}$ Milli-Q ${ }^{\circledR}$ reagent grade water

10 percent Buffered Formalin:

1. $1,620 \mathrm{~mL}$ MFS

2. $620 \mathrm{~mL}$ formaldehyde

3. 6.48 grams $(\mathrm{g})$ Sodium Phosphate Monobasic $\left(\mathrm{NaH}_{2} \mathrm{P}_{4}\right)$ or Potassium Phosphate Monobasic $\left(\mathrm{KH}_{2} \mathrm{PO}_{4}\right)$

4. 10.5 grams $(\mathrm{g})$ Sodium Phosphate Dibasic $\left(\mathrm{Na}_{2} \mathrm{HPO}_{4}\right)$ or Potassium Phosphate Dibasic $\left(\mathrm{K}_{2} \mathrm{HPO}_{4}\right)$

5. $0.75 \mathrm{~mL}$ needed for each replicate. Fill the dispenser.

\subsection{Collection and Preparation of Gametes}

Quality gametes must first be collected, and then diluted to the appropriate concentration for addition to the test vials.

\subsubsection{Selection of Urchins}

1. Take two or three urchins and place inverted individually in shallow empty Carolina bowls. Shake free as much water as possible before placing the urchins in each bowl. 
2. To stimulate release of gametes from gonopores, inject each urchin with 0.5 to $1.0 \mathrm{~mL}$ of a 0.5 molar $(\mathrm{M})$ solution of $\mathrm{KCl}$ in several injections surrounding the soft membrane of the mouth opening. Agitate each urchin slightly after injecting. Gametes should appear from the gonopores within a few minutes. If not, inject with another 0.5 to $1 \mathrm{~mL}$ aliquot of KCL solution. Replace urchins that shed no gametes after two series of injections.

3. Sperm are white to off white in color and eggs are tan to orange in color. If eggs appear, fill the Carolina dish half full with MFS and allow the urchin to shed eggs into the dish while inverted. Collect a few eggs from the bottom of the dish using a $10 \mathrm{~mL}$ disposable syringe with a large gauge blunt-tipped needle attached. Place 2 to 5 drops of eggs onto a scintillation vial containing $10 \mathrm{~mL}$ of filtered seawater. Rinse syringe and repeat for each female. Additional injections of $\mathrm{KCl}$ may be required to collect a full complement of eggs.

4. Select females which have round, well developed eggs, and which do not release clumps of eggs or undeveloped ovarian tissue.

5. If sperm is shed from the urchins, place males upright on a pad made up of several layers of paper towels. Dab each male with additional paper towels to remove as much water as possible from the surface of the urchins. It is important to collect the sperm without any water coming in contact with it.

6. If sperm is watery, reject the animal and choose another. Sperm should be the consistency of condensed milk. Collect sperm using a Pasteur pipette with a rubber bulb attached.

Generally, a gamete check is performed in order to ensure that both the male and the female urchins used in the test have gametes with a high degree of viability. If the gamete check is performed, two to five females (depending on confidence in the proportion of urchins in the holding facility in good reproductive status) and at least two males should be selected using the above procedures. The check is performed by adding 5 to 7 drops of a concentrated dilution of sperm to the eggs in the scintillation vials (collected as described above) and observing the eggs under the microscope after 10 minutes. The concentrated dilution of sperm is usually made by diluting 20-50 microliters $(\mu \mathrm{L})$ of sperm in $10 \mathrm{~mL}$ of filtered seawater. If the proportion of eggs fertilized is high (95-100 percent), that female and male may be used in the pretest and test. Sperm from a number of males or females may be combined in the beginning if the gamete check reveals a number of high quality animals or the confidence is high in the quality of the gametes. Once a good male and female are selected a pretest can be conducted to determine the correct dilution of sperm to use in the test (Attachment B). 


\subsubsection{Obtain Eggs}

1. Place selected female inverted into a small Carolina dish and add enough seawater to cover one half of the urchin's test. Stimulate release of eggs as described above with $\mathrm{KCl}$ injections.

2. Collect eggs as above using the $10 \mathrm{~mL}$ syringe. Remove needle before dispensing eggs into a disposable shell vial or other clean container capable of holding 25-50 mL. Collect enough eggs for pretest and test. If female stops giving eggs, give one or two additional injections of KCL and agitate the urchin and replace inverted into the dish.

3. Add MFS to fill shell vials, gently mixing eggs. Allow eggs to settle to bottom of vial. Remove water with a pipette. Replace water, again gently mixing the eggs.

\subsubsection{Prepare Appropriate Egg Concentration}

1. Put approximately $100 \mathrm{~mL}$ of $30 \%$ MFS in a $250 \mathrm{~mL}$ beaker, and add enough washed eggs to bring the egg density to approximately 10,000 per $\mathrm{mL}$. If more than 400 total replicates (27 treatments) are to be tested, a larger amount of water and a correspondingly larger amount of eggs should be used. Two hundred $\mu \mathrm{L}$ of this egg solution will be used per replicate, and it is easier to maintain proper mixing and uniform egg density if there is an excess of at least 50 percent.

2. Check egg density and adjust to within approximately 9000 to 11,000 eggs per $\mathrm{mL}$, as follows. Gently swirl egg solution until evenly mixed. Using a pipette, add $1 \mathrm{~mL}$ of the solution to a vial containing nine $\mathrm{mL}$ seawater. Mix and transfer $1 \mathrm{~mL}$ of this diluted solution to a second vial containing $4 \mathrm{~mL}$ of seawater. Again, mix and transfer $1 \mathrm{~mL}$ of this diluted solution to a counting slide such as a Sedgewick-Rafter slide.

3. Using a microscope (either a compound microscope with a 10x objective or a dissecting scope may be used here), count the number of eggs on the slide. If the number is not between 180 and 220, then adjust by adding eggs or water. If egg count is greater than 220 use the following formula to calculate the amount of water to add:

(i.) $[($ Egg count -200$) \div 200] \times$ Current volume of eggs $=$ Volume of seawater to add to stock $(\mathrm{mL})$

If egg count is less than 200, add a small amount of eggs. Since it is less arbitrary and more likely to arrive at an acceptable count when using the water addition formula, it is better to originally overestimate the amount of eggs to add to the $100 \mathrm{~mL}$ of water.

4. Repeat steps 2 and 3 until an acceptable egg count (between 180 and 220) is obtained.

\subsubsection{Obtain Sperm}

Place selected $\mathrm{KCl}$ injected males on a pad made up of several layers of paper towels dab each male with additional paper towels to remove as much water as possible from the 
surface of the urchins. Once sperm has contacted water it becomes activated and has a limited life span. This species has a tendency to expel water from the anus during gamete collection so it is important to watch when this happens and discard the sperm on the surface of the urchin at that time, dab with a paper towel to remove water and wetted sperm and continue collecting only dry sperm. It may be necessary to repeatedly invert the urchin for short periods of time or provide additional injections of $\mathrm{KCl}$ to facilitate additional shedding of sperm. Collect about one $\mathrm{mL}$ of unwetted sperm from between spines using a pasteur pipette. Place sperm into a plastic microcentrifuge tube. Keep on ice until used. Do not allow the sperm to freeze. To prevent this, wrap each microcentrifuge tube in a small amount of paper towel before placing it on ice. Be careful not to add any water or sperm which has contacted water to the vials. High quality sperm collected dry and kept on ice will last at least eight hours without measurable decline in viability.

\subsubsection{Prepare Appropriate Sperm Dilution}

It is desirable for control fertilization to be within 60-95 percent. Although controls outside these bounds do not automatically disqualify a test, particularly if a valuable dose response is generated, the sensitivity of the test is reduced by fertilization rates greater than 95 percent and good dose responses may be difficult to obtain with less than 60 percent fertilization in controls. Density of sperm in the sperm solution should be determined with this goal in mind. Condition of the animals and length of acclimation to the aquarium may affect the chosen sperm density. The pretest (Attachment 2) may be used to calculate an appropriate sperm dilution. Due to the sensitivity of $S$. purpuratus sperm to $\mathrm{pHs}$ greater than 8.1, a higher concentration of sperm is generally required in tests utilizing Gulf coast sea waters and pore waters. This effectively reduces the overall sensitivity of the test. Generally, a dilution of between 1:2500 and 1:750 will result in desirable fertilization rates, if the animals are in good condition.

For example, if a sperm dilution of 1:2500 is required (as determined from the pretest), add $20 \mu \mathrm{L}$ sperm to $5 \mathrm{~mL}$ MFS. Mix thoroughly, and add $1 \mathrm{~mL}$ of this solution to $9 \mathrm{~mL}$ of MFS. Sperm should not be wetted until just before starting the test. Sperm wetted more than 10 minutes before the test has begun, including sperm dilutions used in any pretest, should be discarded and a new dilution made from sperm kept on ice.

\subsection{TEST PROCEDURES}

1. Add $50 \mu \mathrm{L}$ appropriately diluted sperm to each vial. Record the time of sperm addition. Sperm should be used within 10 minutes of wetting.

2. Incubate all test vials at $12 \pm 1^{\circ} \mathrm{C}$ for 20 minutes. At this point it is useful to set a timer for five to ten minutes prior to the end of the incubation period. This will notify the worker early enough to be ready to start the next step exactly on time.

3. While gently swirling the egg solution to maintain even mixing of eggs, use a $200 \mu \mathrm{L}$ pipetter to add $200 \mu \mathrm{L}$ diluted egg suspension to each vial. Pipette tips are cut using a clean razor blade to prevent crushing the eggs during pipetting. Record the time of egg addition. 
4. Incubate for 20 minutes at $12 \pm 1^{\circ} \mathrm{C}$. The timer may be used again at this point.

5. Add $0.75 \mathrm{~mL}$ of 10 percent buffered formalin to each sample with a re-pipette bottle.

6. Vials may now be capped and stored overnight or for several days until evaluated. Fertilization membranes are easiest to see while eggs are fairly fresh, so evaluation within two to three days may decrease the time required for evaluation.

\subsection{DATA COLLECTION AND TABULATION}

1. Transfer approximately $1 \mathrm{~mL}$ eggs and water from bottom of test vials to counting slide. Observe eggs using compound microscope under $100 \mathrm{X}$ magnification. Dark field viewing is useful here in identifying fertilization membranes; however, S. purpuratus fertilization membranes are typically very easily discernable.

2. Count 100 eggs/sample using hand counter with multiple keys (such as a blood cell counter), using one key to indicate fertilized eggs and another to indicate unfertilized eggs. Fertilization is defined by the presence of fertilization membrane surrounding egg.

3. Calculate fertilization percentage for each replicate test:

(ii.) $\frac{\text { (Total number of eggs - Number of eggs unfertilized })}{\text { Total number of eggs }} \times 100=$ Percent of eggs fertilized

\subsection{DATA ANALYSIS}

Data are recorded on standardized data sheets (See Attachments C to G). Normally, percent fertilization in each treatment is compared to an appropriate reference treatment (seawater, pore water or sea surface microlayer from an uncontaminated environment). Statistical comparisons are made using analysis of variance (ANOVA) and Dunnett's $t$-test (Sokal and Rohlf, 1981) on the arc sine square root transformed data. For multiple comparisons among treatments, Ryan's Q test (Day and Quinn, 1989) with the arc sine square root transformed data is recommended. The trimmed Spearman-Karber method with Abbott's correction is recommended to calculate $\mathrm{EC}_{50}$ values for dilution series tests (Hamilton and others, 1977)

\subsection{QUALITY CONTROL}

Quality control tests may be run using both positive and negative controls with multiple replicates (as many as desired). Typically, a reference toxicant dilution series (sodium dodecyl sulfate) is tested with each test to evaluate the effectiveness of the sperm dilution chosen. Negative controls may include reference pore water, filtered sea water, and/or reconstituted brine. 


\subsection{TRAINING}

A trainee will conduct the test with supervision initially. Determining egg concentrations and fertilization counts are test specific activities. These functions can be performed independently after a trainee has demonstrated he or she can accurately reproduce the test.

\subsection{SAFETY}

The sea urchin fertilization toxicity test poses little risk to those performing it. Care should be taken when making and dispensing the 10 percent buffered formalin solution; use a hood if available, but make sure the test area is well ventilated. Protective gloves can be worn when pipetting or dispensing formalin or potentially toxic samples.

Care should be taken when collecting or otherwise handling sea urchins. Urchin spines can be sharp and fragile and may puncture the skin and break off if handled roughly. First aid similar to treatment of wood splinters is effective in this case (removal of spine and treatment with antiseptic). Collection of sea urchins by snorkeling should not be done alone.

\subsection{ATTACHMENTS}

Attachment A. Equipment List for Fertilization Toxicity Test

Attachment B. Pretest to Insure Selection of Quality Gametes

Attachment C. Water Quality Adjustment Data Form

Attachment D. Sea Urchin Pretest Data Sheet

Attachment E. Sea Urchin Pretest Continuation Data Sheet

Attachment F. Sea Urchin Fertilization/Embryological Development Toxicity Test Gamete Data Sheet

Attachment G. Sea Urchin Fertilization Toxicity Test Fertilization Data Sheet

\subsection{REFERENCES}

Day, R.W., and Quinn, G.P., 1989, Comparisons of treatments after an analysis of variance in ecology: Ecology Monograph, v. 59, p. 433-463.

Hamilton, M.A., Russo, R.C., and Thurston, R.V., 1977, Trimmed Spearman-Karber method for estimating median lethal concentrations in toxicity bioassays: Environmental Science and Technology, v.11, no. 7, p. 714-719; Correction, v. 12, no. 4, p. 417 (1978).

Sokal, R.R., and Rohlf, F.J., 1981, Biometry: $2^{\text {nd }}$ edition: San Francisco, Ca., W.H. Freeman and Company, 859 p. 
Prepared by:

James M. Biedenbach

Biologist

Approved by:

R. Scott Carr

Field Station Leader

Laverne Cleveland

Chief, Field Stations Research Branch

\author{
Paul Heine \\ Quality Assurance Officer
}




\section{$\underline{\text { Attachment A }}$}

\section{EQUIPMENT LIST FOR FERTILIZATION TOXICITY TEST}

Small Carolina dishes (at least 2)

$20 \mathrm{~mL}$ Wheaton scintillation vials (These should be type shipped with caps with polyseal cone liners. If other brand or type is used, the vials should be tested for toxicity prior to use.)

$400 \mathrm{~mL}$ beaker or wide-mouthed thermos for holding vials of sperm

$250 \mathrm{~mL}$ beakers (4)

Pasteur pipettes and latex bulbs

Plastic microcentrifuge tubes

$25 \mathrm{~mL}$ shell vials or equivalent

Test tube rack (to hold shell vials)

$10 \mathrm{cc}$ syringe with large diameter blunt ended needle (make by grinding sharp point off the needle with a grinding stone)

$1 \mathrm{cc}$ syringe with fine tip needle at least $2 \mathrm{~cm}$ in length.

$0.5 \mathrm{M} \mathrm{KCl}$ solution

Paper towels

Marking pens

Ice

10-100 $\mu \mathrm{L}$ pipetter

50-200 $\mu \mathrm{L}$ pipetter

$5 \mathrm{~mL}$ pipetters $(2)$

Counting slide such as Sedgewick-Rafter chamber

Compound microscope with 10x objective and dark field capability

Hand tally counter

Calculator

Timer for exposure / incubation periods

Buffered formalin and dispenser

Filtered $(0.45 \mu \mathrm{m})$ seawater, adjusted to $30 \%$

Data sheets

Milli -Q ${ }^{\circledR}$ reagent grade water

Approximately $100 \%$ o concentrated brine

Biological control chamber or incubator adjusted to $12^{\circ} \mathrm{C}$ 


\section{$\underline{\text { Attachment B }}$}

\section{PRETEST TO INSURE SELECTION OF QUALITY GAMETES}

1. Using the procedure in section 2.4.1, select 1 to 4 females and at least 1 male urchin to be used in the pretest.

2 Fill pretest vials with $5 \mathrm{~mL}$ of reference water. There should be at least two vials for each combination of male, female, and pretest sperm concentration (step 4 below). For example, in a pretest with 2 females, 1 male, and 5 pretest sperm concentrations, 20 vials ( 2 X 2 X 5 ) would be needed. Additional reference solutions may be added to the pretest to aid in selecting the proper sperm dilution. Typically, one rep each of three different reference toxicant concentrations $(2.5$, 5.0 , and $10 \mathrm{mg} / \mathrm{L}$ ) is added for each sperm concentration to ascertain if the sperm dilution selected will fall within the control range for that reference toxicant. Arrange and mark vials accordingly in a rack and incubate at $12{ }^{\circ} \mathrm{C}$ until the test solutions acclimate to the test temperature.

3. Perform steps 2.4.2 (Egg Collection) and 2.4.3 (Egg Dilution) for each female urchin. Make enough volume of the egg suspension to perform the pretest and the test.

4. Perform step 2.4.4 (Sperm Collection) for each male urchin or male combination. Prepare a dilution series of sperm concentrations which will bracket the 60-95 percent fertilization rate in the test. Sperm dilution will depend on the health and reproductive status of the male urchin, but in most cases the following "standard dilution" should be used:

1: 250 ( $20 \mu \mathrm{L}$ dry sperm added to $5 \mathrm{~mL}$ MFS. This concentration is used only as stock solution to make up the rest of the dilution series and is not used full strength in the pretest.)

1: 1000 ( $1 \mathrm{~mL}$ of $1: 250$ and $3 \mathrm{~mL}$ MFS)

1: 1250 ( $1 \mathrm{~mL}$ of $1: 250$ and $4 \mathrm{~mL}$ MFS)

1: 2500 ( $1 \mathrm{~mL}$ of $1: 250$ and $9 \mathrm{~mL}$ MFS)

1: 5000 ( $2 \mathrm{~mL}$ of $1: 2500$ and $2 \mathrm{~mL}$ MFS)

1: 7500 (2 $\mathrm{mL}$ of 1:2500 and $4 \mathrm{~mL}$ MFS)

Sperm must be used within 10 minutes of dilution and should be made up in MFS maintained at the test temperature. Leave undiluted sperm on ice and retain, because a new sperm dilution of the concentration determined in this pretest will be needed for the toxicity test. Sperm diluted for use in the pretest may not be used in the toxicity test, because the time elapsed since the addition of water is too great.

5. As in section 3.0 add $50 \mu \mathrm{L}$ of the diluted sperm to each pretest vial. Incubate for 20 minutes at approximately $12{ }^{\circ} \mathrm{C}$, and add $200 \mu \mathrm{L}$ of the egg suspension. Incubate for another 20 minutes, then fix with $1 \mathrm{~mL}$ of the buffered formalin solution. 


\section{$\underline{\text { Attachment B. Continued }}$}

6. As in section 4.0, obtain a fertilization rate for the vials. There is no need to count all vials, enough vials should be counted to determine a good male/female combination, and an appropriate sperm dilution factor. If more than one male/female combination is acceptable, this is a good opportunity to choose a female which exhibits easily visible fertilization membranes or in cases where there are many samples, to combine eggs from different females. The appearance of the fertilization membranes may vary among female urchins, and presence of easily visible membranes facilitates counting. 


\section{Attachment C}

\section{WATER QUALITY ADJUSTMENT DATA FORM}

STUDY PROTOCOL

SAMPLE DESIGNATION
INITIALS

DATE

A. Salinity Adjustment:

Initial volume $(\mathrm{mL})$

Initial salinity (\%o)

Vol. Milli-Q ${ }^{\circledR}$ water added (mL)

Vol. _ \% $\%$ brine added (mL)

Percent of original sample

(initial volume/final volume $\times 100$ )

B. Character of Sample (after salinity adjustment):

Volume (mL)

Salinity (\%o)

$\mathrm{pH}$

Dissolved oxygen $(\mathrm{mg} / \mathrm{L})$

DO saturation (percent)

Total ammonia (mg/L)

Sulfide (mg/L)

COMMENTS 


\section{$\underline{\text { Attachment D }}$}

\section{SEAURCHIN PRETEST DATA SHEET}

\begin{tabular}{lr}
\hline STUDY PROTOCOL INITIALS \\
SAMPLE DESIGNATION_ DATE_
\end{tabular}

\section{$\underline{\text { EGGS }}$}

Female number:

Collection time:

Count:

\section{SPERM}

Male number:

Collection time:

Dilution start time:

\section{TEST TIMES}

Sperm in:

Eggs in:

Formalin in:

\section{SPERM DILUTION:}

COMMENTS:

PERCENT FERTILIZATION Reference sample designation:

Female \#

Sperm Dilution

$\overline{\overline{(20}}$

$\overline{\overline{ }}$

$\overline{\overline{(n+1}}$ Male \#

$\underline{\text { REP } 1} \quad \underline{\text { REP } 2} \underline{\text { REP } 4}$

PERCENT FERTILIZATION Reference sample designation:

Female \# Male \#

Sperm dilution

$\begin{array}{lll}\underline{\text { REP 1 }} & \underline{\underline{R E P} 2} & \underline{\text { REP 3 }} \\ \square & - & - \\ \square & - & -\end{array}$




\section{$\underline{\text { Attachment E }}$}

\section{SEA URCHIN PRETEST CONTINUATION DATA SHEET}

STUDY PROTOCOL

SAMPLE DESIGNATION
INITIALS

DATE

PERCENT FERTILIZATION Reference sample designation:

Female \# Male \#

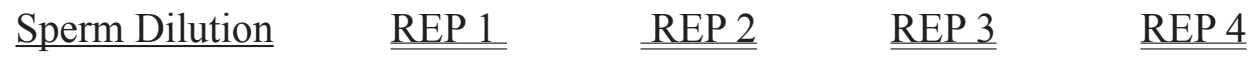

$\overline{\overline{ }} \overline{\overline{ }} \overline{\overline{ }}$

PERCENT FERTILIZATION Reference sample designation:

Female \# Male \#

$\underline{\text { Sperm dilution }}$

$\begin{array}{ll}\underline{\text { REP 1 }} & \underline{\text { REP 2 }} \\ - & - \\ - & - \\ - & -\end{array}$

$\underline{\underline{R E P} 3}$

$\underline{\underline{\text { REP } 4}}$

$\overline{\overline{ }}$

PERCENT FERTILIZATION Reference sample designation:

Female \# Male \#

$\underline{\text { Sperm Dilution }}$

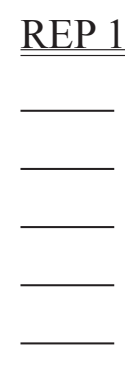

$\underline{\underline{R E P} 3}$

$\underline{\text { REP } 4}$ 


\section{$\underline{\text { Attachment F }}$}

\section{SEA URCHIN FERTILIZATION/EMBRYOLOGICAL DEVELOPMENT TOXICITY TEST GAMETE DATA SHEET}

STUDY PROTOCOL

SAMPLE DESIGNATION
INITIALS

DATE

\section{EGGS}

Collection time:

Initial count/volume:

Final count:

\section{SPERM}

Collection time:

Dilution start time:

Sperm dilution:

Test start temperature:

\section{TEST TIMES}

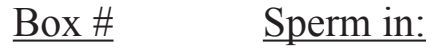

\section{COMMENTS}

Eggs in: $\quad$ Formalin in:

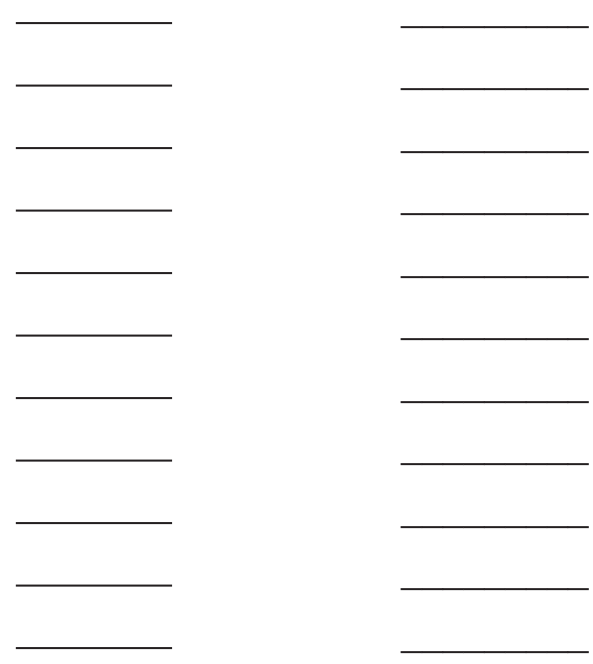




\section{$\underline{\text { Attachment G }}$}

\section{SEA URCHIN FERTILIZATION TOXICITY TEST FERTILIZATION DATA SHEET}

STUDY PROTOCOL

SAMPLE DESIGNATION
INITIALS

DATE

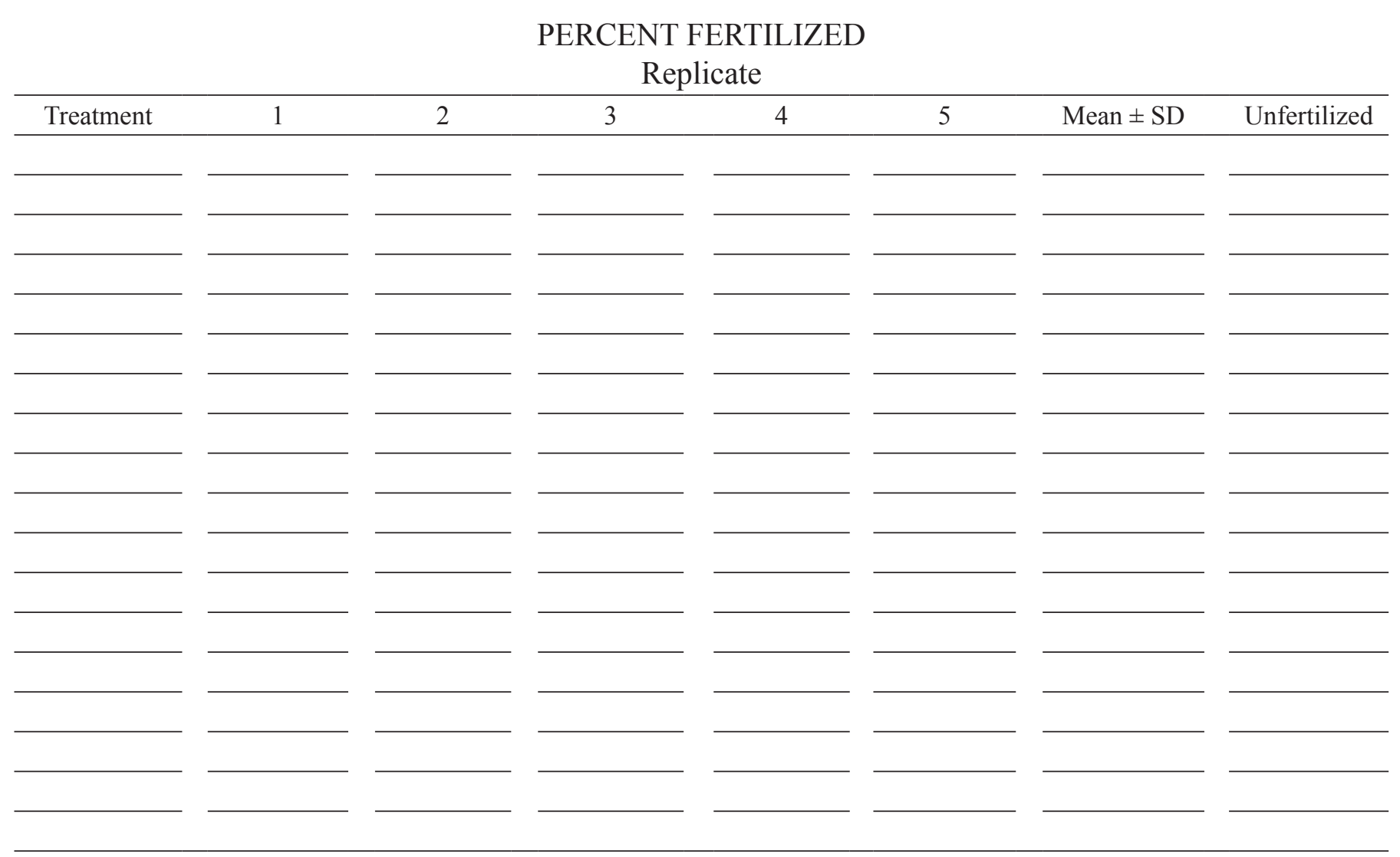

\section{COMMENTS}


Appendix 8. Sodium dodecyl sulfate reference toxicant control EC50s from toxicity tests with Strongylocentrotus purpuratus from 1997-2009.

[EC50, Effective concentration 50 percent in milligrams per liter; na, not applicable; ${ }^{\circ} \mathrm{C}$, degrees Celsius; PS ref tox, Puget Sound Reference Toxicity Test; PSAMP, Puget Sound Assessment and Monitoring Program; comp, comparison]

\begin{tabular}{|c|c|c|c|c|c|}
\hline Year & Test & EC50 & EC50 & Method $^{1}$ & Stations included \\
\hline 1997 & Puget Sound-1 & 2.41 & na & $30 / 30$ exposure, $15^{\circ} \mathrm{C}$ & $26-58,83-100$ \\
\hline 1997 & Puget Sound-2A & 3.23 & na & $30 / 30$ exposure, $15^{\circ} \mathrm{C}$ & $59-82$ \\
\hline 1997 & Puget Sound-4 & 3.51 & na & $30 / 30$ exposure, $15^{\circ} \mathrm{C}$ & $1-25$ \\
\hline 1997 & PS ref tox & 2.91 & na & $30 / 30$ exposure, $15^{\circ} \mathrm{C}$ & None \\
\hline 1998 & Puget Sound II-2 & 2.32 & na & $30 / 30$ exposure, $15^{\circ} \mathrm{C}$ & $157-205,114,115$ \\
\hline 1998 & Puget Sound II-3 & 5.36 & na & $30 / 30$ exposure, $15^{\circ} \mathrm{C}$ & $106-132$ \\
\hline 1998 & Puget Sound II-4 & 4.03 & na & $30 / 30$ exposure, $15^{\circ} \mathrm{C}$ & $133-156$ \\
\hline 1999 & Puget Sound III-2A & 3.69 & na & $30 / 30$ exposure, $15^{\circ} \mathrm{C}$ & $254-305$ \\
\hline 1999 & Puget Sound III-2B & 2.31 & na & $30 / 30$ exposure, $15^{\circ} \mathrm{C}$ & $206-253$ \\
\hline 2002 & PSAMP 2002 & 4.77 & na & $30 / 30$ exposure, $15^{\circ} \mathrm{C}$ & $1-161$ \\
\hline 2002 & PSAMP 2002 & 5.70 & na & $30 / 30$ exposure, $15^{\circ} \mathrm{C}$ & $177-2123$ \\
\hline 2003 & PSAMP 2003 & na & 2.49 & $20 / 20$ exposure, $12^{\circ} \mathrm{C}$ & $297-523$ \\
\hline 2003 & PSAMP 2003 & na & 2.76 & $20 / 20$ exposure, $12^{\circ} \mathrm{C}$ & $527-1387$ \\
\hline 2005 & PSAMP 2004 & 3.69 & na & $20 / 20$ exposure, $12^{\circ} \mathrm{C}$ & $8-336$ \\
\hline 2005 & PSAMP 2004 & na & 3.54 & $20 / 20$ exposure, $12^{\circ} \mathrm{C}$ & 24-336 (selected) \\
\hline 2005 & PSAMP 2003 retest & 5.70 & na & $20 / 20$ exposure, $12^{\circ} \mathrm{C}$ & $297-1387$ \\
\hline 2006 & PSAMP methods comp & na & 3.58 & $20 / 20$ exposure, $12^{\circ} \mathrm{C}$ & All \\
\hline 2006 & PSAMP methods comp & 3.49 & na & $20 / 20$ exposure, $12^{\circ} \mathrm{C}$ & All \\
\hline 2006 & PSAMP test 1 & na & 3.66 & $20 / 20$ exposure, $12^{\circ} \mathrm{C}$ & $3-165$ \\
\hline 2006 & PSAMP test 2 & na & 3.87 & $20 / 20$ exposure, $12^{\circ} \mathrm{C}$ & $189-667$ \\
\hline 2007 & PSAMP test 1 & na & 2.62 & $20 / 20$ exposure, $12^{\circ} \mathrm{C}$ & $5-151$ \\
\hline 2007 & PSAMP test 2 & na & 3.54 & $20 / 20$ exposure, $12^{\circ} \mathrm{C}$ & $155-371$ \\
\hline 2007 & Urban waters & na & 2.42 & $20 / 20$ exposure, $12^{\circ} \mathrm{C}$ & All \\
\hline 2008 & PSAMP & na & 3.56 & $20 / 20$ exposure, $12^{\circ} \mathrm{C}$ & All \\
\hline 2008 & Urban waters & na & 2.83 & $20 / 20$ exposure, $12^{\circ} \mathrm{C}$ & All \\
\hline 2009 & PSAMP test 1 & na & 3.54 & $20 / 20$ exposure, $12^{\circ} \mathrm{C}$ & $002-234$ \\
\hline 2009 & PSAMP test 2 & na & 3.54 & $20 / 20$ exposure, $12^{\circ} \mathrm{C}$ & $239-498$ \\
\hline \multirow[t]{3}{*}{2009} & Urban waters & na & 3.08 & $20 / 20$ exposure, $12^{\circ} \mathrm{C}$ & All \\
\hline & Mean & 3.79 & 3.22 & & \\
\hline & Standard deviation & 1.19 & .50 & & \\
\hline
\end{tabular}

${ }^{1}$ Refers to minutes of exposure of sperm and then egg with sperm at incubated temperature. 
Appendix 9. Aransas Bay negative control 100-percent pore-water and Millipore ${ }^{\circledR}$ filtered seawater (MFS) dilution water means and standard deviations from toxicity tests with Strongylocentrotus purpuratus from 1997-2009.

[SD, standard deviation; MFS, Millipore ${ }^{\circledR}$ filtered seawater; na, not applicable; PS ref tox, Puget Sound Reference Toxicity Test; PSAMP, Puget Sound Assessment and Monitoring Program]

\begin{tabular}{|c|c|c|c|c|c|c|}
\hline Year & Test & $\begin{array}{c}\text { Negative } \\
\text { control mean }\end{array}$ & SD & $\begin{array}{l}\text { MFS } \\
\text { mean }\end{array}$ & SD & $\begin{array}{l}\text { Stations } \\
\text { included }\end{array}$ \\
\hline 1997 & Puget Sound-1 & $\mathrm{na}^{1}$ & $\mathrm{na}^{1}$ & 96.2 & 3.1 & $26-58,83-100$ \\
\hline 1997 & Puget Sound-2A & $\mathrm{na}^{1}$ & $\mathrm{na}^{1}$ & 91.8 & 5.7 & $59-82$ \\
\hline 1997 & Puget Sound-4 & $\mathrm{na}^{1}$ & $\mathrm{na}^{1}$ & 92.6 & 3.9 & $1-25$ \\
\hline 1997 & PS ref tox & $\mathrm{na}^{1}$ & $\mathrm{na}^{1}$ & 85.5 & 4.8 & None \\
\hline 1998 & Puget Sound II-2 & $\mathrm{na}^{1}$ & $\mathrm{na}^{1}$ & 79.0 & 4.9 & $157-205,114,115$ \\
\hline 1998 & Puget Sound II-3 & $\mathrm{na}^{1}$ & $\mathrm{na}^{1}$ & 97.3 & 2.0 & $106-132$ \\
\hline 1998 & Puget Sound II-4 & $\mathrm{na}^{1}$ & $\mathrm{na}^{1}$ & 98.7 & 1.7 & $133-156$ \\
\hline 1999 & Puget Sound III-2A & $\mathrm{na}^{1}$ & $\mathrm{na}^{1}$ & 96.4 & 2.4 & $254-305$ \\
\hline 1999 & Puget Sound III-2B & $\mathrm{na}^{1}$ & $\mathrm{na}^{1}$ & 96.9 & 2.1 & $206-253$ \\
\hline 2002 & PSAMP 2002 & 98.8 & 1.3 & 99.0 & 1.4 & $1-161$ \\
\hline 2002 & PSAMP 2002 & 99.0 & 1.0 & 99.5 & .8 & $177-2123$ \\
\hline 2003 & PSAMP 2003 & 93.6 & 4.0 & 92.3 & 3.2 & $297-523$ \\
\hline 2003 & PSAMP 2003 & 94.8 & 1.5 & 95.2 & 2.6 & $527-1387$ \\
\hline 2005 & PSAMP 2004 & 94.2 & 3.2 & 93.1 & 6.9 & $8-336$ \\
\hline 2005 & PSAMP 2004 & 95.4 & 1.8 & 96.9 & .8 & 24-336 (selected) \\
\hline 2005 & PSAMP 2003 retest & 99.2 & .6 & 98.5 & 1.2 & $297-1387$ \\
\hline 2006 & PSAMP test 1 & 92.4 & 4.3 & 98.8 & .8 & $3-165$ \\
\hline 2006 & PSAMP test 2 & 89.8 & 3.4 & 95.2 & 4.8 & $189-667$ \\
\hline 2007 & PSAMP test 1 & 95.3 & 3.5 & 85.9 & 7.6 & $5-151$ \\
\hline 2007 & PSAMP test 2 & 92.4 & 2.7 & 94.6 & 2.5 & $155-371$ \\
\hline 2007 & Urban waters & 97.3 & 2.3 & 93.3 & 5.7 & All \\
\hline 2008 & PSAMP & 99.6 & .7 & 99.5 & .7 & All \\
\hline 2008 & Urban waters & 98.3 & 2.1 & 96.4 & 2.0 & All \\
\hline 2009 & PSAMP test 1 & 99.3 & .7 & 98.5 & .5 & $002-234$ \\
\hline 2009 & PSAMP test 2 & 99.4 & .7 & 99.0 & .9 & $239-498$ \\
\hline \multirow[t]{3}{*}{2009} & Urban waters & 82.0 & 5.3 & 86.7 & 9.1 & All \\
\hline & Mean & 95.7 & & 94.5 & & \\
\hline & Standard deviation & 3.1 & & 5.1 & & \\
\hline
\end{tabular}

\footnotetext{
${ }^{1}$ A different control pore water from Redfish Bay was used for these tests.
} 
Publishing support provided by: Rolla Publishing Service Center

For more information concerning this publication, contact: Director, USGS Columbia Environmental Research Center 4200 New Haven Road

Columbia, MO 65201

(573) 875-5399

Or visit the Columbia Environmental Research Center Web site at: http://www.cerc.usgs.gov 



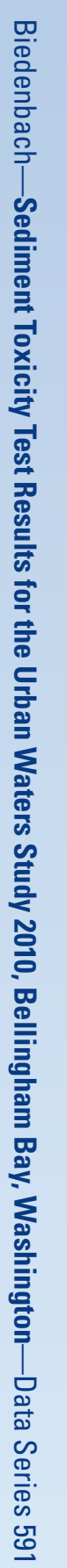

